\title{
Binarity among CEMP-no stars: an indication of multiple formation pathways? ${ }^{\star, \star \star}$
}

\author{
A. Arentsen ${ }^{1}$, E. Starkenburg ${ }^{1}$, M. D. Shetrone ${ }^{2}$, K. A. Venn ${ }^{3}$,É. Depagne ${ }^{4}$, and A. W. McConnachie ${ }^{5}$ \\ 1 Leibniz-Institut für Astrophysik Potsdam (AIP), An der Sternwarte 16, 14482 Potsdam, Germany \\ e-mail: aarentsen@aip.de \\ 2 McDonald Observatory, University of Texas at Austin, HC75 Box 1337-MCD, Fort Davis, TX 79734, USA \\ 3 Department of Physics \& Astronomy, University of Victoria, Victoria, BC V8W 3P2, Canada \\ ${ }^{4}$ South African Astronomical Observatory (SAAO), Observatory Road Observatory Cape Town, WC 7925, South Africa \\ 5 NRC Herzberg Institute of Astrophysics, 5071 West Saanich Road, Victoria, BC V9E 2E7, Canada
}

Received 28 August 2018 / Accepted 4 November 2018

\begin{abstract}
Carbon-enhanced metal-poor (CEMP) stars comprise a high percentage of stars at the lowest metallicities. The stars in the CEMP-no subcategory do not show any $s$-process enhancement and therefore cannot easily be explained by transfer of carbon and $s$-process elements from a binary AGB companion. We have performed radial velocity monitoring of a sample of 22 CEMP-no stars to further study the role that binarity plays in this type of CEMP star. We find four new binary CEMP-no stars based on their radial velocity variations; this significantly enlarges the population of known binaries to a total of 11 . One of the new stars found to be in a binary system is HE 0107-5240, which is one of the most iron-poor stars known. This supports the binary transfer model for the origin of the abundance pattern of this star. We find a difference in binary fraction in our sample that depends on the absolute carbon abundance, with a binary fraction of $47_{-14}^{+15} \%$ for stars with a higher absolute carbon abundance and $18_{-9}^{+14} \%$ for stars with a lower absolute carbon abundance. This might imply a relation between a high carbon abundance and the binarity of a metal-poor star. Although binarity does not equate to mass transfer, there is a possibility that a CEMP-no star in a binary system has been polluted, and care has to be taken in the interpretation of their abundance patterns. We furthermore demonstrate the potential of Gaia of discovering additional binary candidates.
\end{abstract}

Key words. stars: chemically peculiar - binaries: spectroscopic - stars: AGB and post-AGB - Galaxy: halo - galaxies: formation

\section{Introduction}

To study the earliest times in the Universe, we do not have to go to high redshift. Our Milky Way still hosts remnants from these early times in the form of extremely metal-poor stars that are expected to be almost as old as the Universe, and we can study them in detail. At the lowest metallicities, the fraction of stars enhanced in carbon increases dramatically (Beers et al. 1992; Norris et al. 1997). These carbon-enhanced metal-poor (CEMP) stars comprise 15-20\% of the very metal-poor stars $\left([\mathrm{Fe} / \mathrm{H}]^{1}<-2.0\right)$, which increases to $\sim 40 \%$ for extremely metalpoor stars $([\mathrm{Fe} / \mathrm{H}]<-3.0)$ and even higher percentages at lower metallicities (Yong et al. 2013; Lee et al. 2013; Placco et al. 2014).

Different types of CEMP stars have initially been defined by Beers \& Christlieb (2005). The two main classes are CEMP- $s$ stars, which show additional enhancement in $s$-process elements (with $[\mathrm{C} / \mathrm{Fe}]>+0.7$ and $[\mathrm{Ba} / \mathrm{Fe}]>+1.0$ ), and the CEMP-no stars, which do not show any $s$-process enhancement and usually occur at lower metallicities (with $[\mathrm{C} / \mathrm{Fe}]>+0.7$ and

\footnotetext{
* Based on observations made with the Southern African Large Telescope (SALT) and the Canada-France-Hawaii Telescope (CFHT).

$\star \star$ Full Table B.2 is only available at the CDS via anonymous ftp to cdsarc.u-strasbg. fr (130.79.128.5) or via http://cdsarc. u-strasbg.fr/viz-bin/qcat?J/A+A/621/A108

${ }_{1}[\mathrm{X} / \mathrm{Y}]=\log \left(N_{\mathrm{X}} / N_{\mathrm{Y}}\right)_{*}-\log \left(N_{\mathrm{X}} / N_{\mathrm{Y}}\right)_{\odot}$, where the asterisk subscript refers to the considered star, and $N$ is the number density.
}

$[\mathrm{Ba} / \mathrm{Fe}]<0.0)$. A subclass of the CEMP- $s$ stars are the CEMP$r / s$ stars, which are additionally enhanced in $r$-process elements. It was reported by Spite et al. (2013) that CEMP stars seemed to occupy two bands in absolute carbon versus metallicity space. The more metal-rich CEMP stars have higher absolute carbon clustering around an absolute carbon abundance $\mathrm{A}(\mathrm{C})^{2} \sim 8.25$, and they turned out to be mainly CEMP- $s$ stars, whereas the more metal-poor CEMP stars are located at a lower $\mathrm{A}(\mathrm{C}) \sim 6.5$, and they are mainly CEMP-no stars. Larger samples of CEMP stars have confirmed this trend (e.g. Bonifacio et al. 2015; Hansen et al. 2015), although there are always some outliers.

Through radial velocity monitoring, it was found that the CEMP- $s$ stars are almost always in a binary system (e.g. McClure \& Woodsworth 1990; Preston \& Sneden 2001; Lucatello et al. 2005; Hansen et al. 2016a), while the CEMP-no stars more often appear to be single stars (Norris et al. 2013a; Starkenburg et al. 2014; Hansen et al. 2016b, afterwards S14 and H16a). CEMP- $s$ stars are thought to have received their carbon and $s$-process elements through mass-transfer from an evolved companion that has gone through the asymptotic giant branch (AGB) phase (Abate et al. 2015).

The exact origin of the CEMP-no stars is not yet clear. CEMP-no stars are not generally considered to be in binary

$2 \mathrm{~A}(\mathrm{X})=\log \epsilon_{\mathrm{X}}=\log \left(N_{\mathrm{X}} / N_{\mathrm{H}}\right)+12$. Throughout this paper, we assume the Asplund et al. (2009) solar abundances. 
systems, but the data indicate that at least some of them are: $\sim 17 \%$ of the sample in H16a. This is close to the binary frequency of $16 \% \pm 4 \%$ found by Carney et al. (2003) for 91 carbonnormal metal-poor $([\mathrm{Fe} / \mathrm{H}] \leq-1.4)$ field red giants. Since most of the CEMP-no stars do not have a binary companion, it is often assumed that the carbon abundance in these stars is intrinsic and therefore reflects the composition of the gas out of which they are formed. The 13 stars with the lowest known metallicities $([\mathrm{Fe} / \mathrm{H}]<-4.5)$ are all CEMP stars, with two exceptions from Caffau et al. (2011) and (likely) Starkenburg et al. (2018). Moreover, most of them do not show clear signatures of $s$-process enhancement. This combination of the most metal-poor stars being enhanced in carbon and not in $s$-process elements suggests that their abundances may be "original". The CEMP-no stars may be early-generation stars born from gas that was polluted by the first generation(s) of massive stars.

One of the possible progenitors of carbon in the early universe are the so-called spinstars (e.g. Meynet et al. 2006, 2010; Chiappini 2013). These are rapidly rotating massive ultra metalpoor stars with strong winds, and they can form large amounts of carbon. Another possibility for the progenitors of carbon are the so-called faint supernovae with mixing-and-fallback models (Umeda \& Nomoto 2003, 2005; Nomoto et al. 2013; Tominaga et al. 2014), in which a supernova does not have sufficient energy to eject all its material into its surroundings, but only the outer layers with the lightest elements are ejected, while the inner part falls back onto the neutron star or black hole at the centre. Yoon et al. (2016) recently suggested that there are two types of CEMP-no stars based on their absolute carbon abundance; they might correspond to the two different progenitors.

It is also possible that some CEMP-no stars have been polluted by a companion, but the binary fraction of CEMP-no stars is not well constrained so far. Studying the CEMP-no binary fraction and binary properties of the population provides us with more information on star formation processes at early times. Additionally, knowledge about the binarity of each individual CEMP-no star is important because it may aid the interpretation of the chemical properties of the star. Key in determining the binarity of stars is radial velocity monitoring, a laborious effort. In this paper we present the results of a large radial velocity monitoring program for CEMP-no stars. The initial sample (described in S14) has been extended with additional spectra for 22 CEMP-no stars, including nine new stars that are not in S14 or H16a, which are mainly located in the southern hemisphere.

This paper is organised as follows. The new observations from this work are described in Sect. 2. In Sect. 3 we present the results of the radial velocity monitoring, and in Sect. 4 we summarise the properties of the CEMP-no binary population. We discuss the results in Sect. 5, give an outlook about what can be achieved with Gaia in Sect. 6, and our conclusions are briefly summarised in Sect. 7.

\section{Data}

\subsection{Sample selection and observations}

We monitored 22 CEMP-no stars for radial velocity variations. The stars were chosen to be additional follow-up for Starkenburg et al. (2014) and to extend the sample to the southern hemisphere. Stars were originally selected from Norris et al. (2013a), and then the sample was expanded with additional stars from different literature sources. The list of targeted stars with some of their properties can be found in Table 1. All stars in our sample have $[\mathrm{Fe} / \mathrm{H}]<-2.5$, and more than $80 \%$ of the sample has $[\mathrm{Fe} / \mathrm{H}]<-3.0$. All stars meet the CEMP criterion $[\mathrm{C} / \mathrm{Fe}] \geq+0.7$, and more than $70 \%$ of the sample meets the stricter CEMP criterion of $[\mathrm{C} / \mathrm{Fe}] \geq+1.0$. Almost all stars satisfy the classical CEMP-no criterion by having $[\mathrm{Ba} / \mathrm{Fe}] \leq 0.0$, within the uncertainties. Two notable exceptions are the hyper metalpoor stars HE 0107-5240 and HE 1327-2326, whose upper limits on $[\mathrm{Ba} / \mathrm{Fe}]$ are higher than +0.9 . However, Matsuno et al. (2017) have revised the CEMP-no definition, taking into account the increasing trend in $[\mathrm{Ba} / \mathrm{Fe}]$ with $[\mathrm{Fe} / \mathrm{H}]$ among CEMP- $s$ stars. The new definition is different from the classical definition for stars with $[\mathrm{C} / \mathrm{Fe}]>+2$, where in this region stars are classified as CEMP-no if they have $[\mathrm{Ba} / \mathrm{C}]<-2$. Both HE 01075240 and HE 1327-2326 satisfy this revised CEMP-no criterion. Additionally, Norris et al. (2013a) pointed out that other chemical properties for these stars are consistent with being CEMPno. One other star that does not satisfy even the new CEMP-no criterion is $\operatorname{SDSS} 0140+2344([\mathrm{Ba} / \mathrm{Fe}]<+0.34)$. This star has an absolute carbon abundance of 5.6, however, which is much lower than the typical value for CEMP- $s$ stars. It is therefore more likely to be CEMP-no.

Between August 2013 and April 2015, we gathered a total of 98 high-resolution spectra with a low signal-to-noise ratio $(\mathrm{S} / \mathrm{N})$ of our target stars, plus spectra of radial velocity standard stars. We made use of the Echelle SpectroPolarimetric Device for the Observation of Stars (ESPaDOnS, Donati et al. 2003; Manset \& Donati 2003) at the Canada-France-Hawaii Telescope (CFHT), with a resolving power of $R \sim 68000$ covering the wavelength range $370-1050 \mathrm{~nm}$. The other spectrograph we used was the High Resolution Spectrograph (HRS, Bramall et al. 2010, 2012; Crause et al. 2014) at the Southern African Large Telescope (SALT, Buckley et al. 2006), which we used in its low-resolution mode with $R \sim 16000$. Our observations started during commissioning of HRS. The HRS is a fibre-fed dualbeam white-pupil echelle spectrograph that yields two spectra: a blue arm that covers from $370-550 \mathrm{~nm}$, and a red arm that covers from 550-890 nm. The number of observed spectra per star for each telescope is indicated in Table 1.

The stars that were observed with CFHT largely overlap with the sample of H16a, which we could not coordinate because our programs were executed at around the same time. We typically have a shorter baseline and a more heterogeneous sample of measurements.

\subsection{Data reduction}

The CFHT spectra were reduced using the dedicated software package LIBRE-ESPRIT (Donati et al. 1997), which includes an automatic wavelength correction using telluric lines. We used the normalised spectra in our radial velocity measurements.

For the SALT data we have adopted the results of the standard HRS pipeline for the spectra taken after commissioning of HRS was completed in late 2013 ( $80 \%$ of the sample). The spectra obtained during commissioning (eight science observations in total) were reduced with the standard IRAF ${ }^{3}$ reduction scripts from the ECHELLE package. These shared-risk spectra had to be addressed individually and were not well suited for reduction with the preliminary HRS pipeline at that time.

\footnotetext{
3 IRAF (Image Reduction and Analysis Facility) is distributed by the National Optical Astronomy Observatories, which are operated by the Association of Universities for Research in Astronomy, Inc., under con-
} tract with the National Science Foundation. 
Table 1. The 22 program stars.

\begin{tabular}{|c|c|c|c|c|c|c|c|c|c|c|c|}
\hline Name & & & $\begin{array}{r}V \\
\text { (mag) }\end{array}$ & $\begin{array}{l}\text { RA } \\
(\mathrm{J} 2000)\end{array}$ & $\begin{array}{l}\text { Dec } \\
(\mathrm{J} 2000)\end{array}$ & {$[\mathrm{Fe} / \mathrm{H}]$} & {$[\mathrm{C} / \mathrm{Fe}]^{b}$} & $\mathrm{~A}(\mathrm{C})^{b}$ & {$[\mathrm{Ba} / \mathrm{Fe}]$} & Ref. & Bin? \\
\hline $\mathrm{BD}+44^{\circ} 493$ & $\mathrm{C}$ & 5 & 9.1 & 022649.7 & +445747 & -3.83 & 1.35 & 5.95 & -0.60 & 2 & 1 \\
\hline BS 16929-005 & $\mathrm{C}$ & 4 & 13.6 & 130329.5 & +335109 & -3.34 & 0.99 & 6.09 & -0.41 & 1 & 1 \\
\hline CS 22878-027 & $\mathrm{C}$ & 4 & 14.4 & 163735.9 & +102208 & -2.51 & $0.86^{d}$ & $6.78^{d}$ & $<-0.75$ & 1 & 1 \\
\hline CS 22949-037 & $\mathrm{C}$ & 5 & 14.4 & 232629.8 & -023958 & -4.38 & 1.16 & 5.97 & -0.60 & 3 & 1 \\
\hline CS 22957-027 & $\mathrm{C}$ & 5 & 13.6 & 235913.1 & -035348 & -3.19 & 2.61 & 7.87 & -0.81 & 3 & 2 \\
\hline CS 29498-043 & $\mathrm{S}$ & 4 & 13.7 & 210352.1 & -294250 & -3.87 & 2.75 & 7.62 & -0.49 & 3 & 1 \\
\hline CS 29502-092 & $\mathrm{C}$ & 6 & 11.9 & 222236.0 & -013828 & -3.30 & 1.06 & 6.59 & -1.36 & 3 & 1 \\
\hline HE 0057-5959 & $\mathrm{S}$ & 2 & 15.8 & 005954.1 & -594330 & -4.08 & 0.86 & 5.21 & -0.46 & 1 & \\
\hline HE 0107-5240 & $\mathrm{S}$ & 4 & 15.1 & 010929.2 & -522434 & -5.44 & 3.97 & 7.03 & $<+0.93$ & 4 & 3 \\
\hline HE $0557-4840$ & $\mathrm{~S}$ & 3 & 15.5 & 055839.3 & -483957 & -4.73 & 1.59 & 5.29 & $<+0.07$ & 5 & \\
\hline HE $1012-1540$ & $\mathrm{C} / \mathrm{S}$ & $5 / 1$ & 14.0 & 101453.5 & -155553 & -4.17 & 2.40 & 6.67 & -0.28 & 3 & 1 \\
\hline HE $1150-0428$ & $\mathrm{C}$ & 7 & 14.9 & 115306.6 & -044503 & -3.47 & 2.37 & 7.35 & -0.48 & 1 & 2 \\
\hline HE $1201-1512^{e}$ & $\mathrm{C} / \mathrm{S}$ & $4 / 2$ & 13.8 & 120337.1 & -152932 & -3.86 & 1.14 & 5.71 & $<+0.05$ & 1 & 1 \\
\hline HE $1300+0157$ & $\mathrm{C}$ & 4 & 14.1 & 130256.2 & +014152 & -3.75 & 1.31 & 5.99 & $<-0.85$ & 1 & 1 \\
\hline HE 1327-2326 & $\mathrm{S}$ & 3 & 13.6 & 133005.9 & -234150 & -5.71 & 4.18 & 6.90 & $<+1.39$ & 6 & 1 \\
\hline HE 1506-0113 & $\mathrm{C} / \mathrm{S}$ & $7 / 1$ & 14.4 & 150914.3 & -012457 & -3.54 & 1.47 & 6.38 & -0.80 & 1 & 2 \\
\hline HE 2139-5432 & $\mathrm{S}$ & 2 & 15.4 & 214242.5 & -541843 & -4.02 & 2.59 & 7.01 & $<-0.33$ & 1 & 3 \\
\hline HE 2142-5656 & $\mathrm{S}$ & 3 & 13.7 & 214620.5 & -564218 & -2.87 & 0.95 & 6.61 & -0.63 & 1 & \\
\hline HE 2202-4831 & $\mathrm{S}$ & 2 & 15.6 & 220606.0 & -481653 & -2.78 & 2.41 & 8.08 & -1.28 & 1 & \\
\hline HE 2247-7400 & $\mathrm{S}$ & 1 & 13.3 & 225119.6 & -734421 & -2.87 & 0.70 & 6.58 & -0.94 & 1 & \\
\hline SDSS J0140+2344 ${ }^{e, f}$ & $\mathrm{C}$ & 4 & 15.1 & 014036.2 & +234458 & -4.00 & 1.13 & 5.56 & $<+0.34$ & 1 & 3 \\
\hline SDSS J1422+0031 & $\mathrm{S}$ & 3 & 16.3 & 142237.4 & +003105 & -3.03 & 1.70 & 7.11 & -1.18 & 7 & 3 \\
\hline
\end{tabular}

Notes. ${ }^{(a)}$ Number of radial velocity measurements added in this program observed with CFHT (C) or SALT (S). ${ }^{(b)}$ Values corrected for evolutionary status, as reported in Yoon et al. (2016). ${ }^{(c)} 1=$ single, $2=$ binary, from Preston \& Sneden (2001), S14 and H16a, $3=$ binary from this work. ${ }^{(d)}$ This star is not in the Yoon et al. (2016) compilation, we report the [C/Fe] from Yong et al. (2013; without evolutionary correction, which is fine since this is not an evolved star), and we computed the $\mathrm{A}(\mathrm{C})$ using the Asplund et al. (2009) solar carbon abundance. ${ }^{(e)}$ These are the dwarf solutions. ${ }^{(f)}$ Sometimes named 53327-2044-515 (e.g. in Yong et al. 2013), but here we use its official SDSS name.

References. (1) Yong et al. (2013), (2) Ito et al. (2013), (3) Roederer et al. (2014), (4) Christlieb et al. (2004), (5) Norris et al. (2007), (6) Frebel et al. (2008), and (7) Aoki et al. (2013).

\subsection{Radial velocity determination}

We determined radial velocities using the IRAF FXCOR package. This package Fourier cross-correlates the observed spectrum with a template spectrum, measuring the relative shift between the two spectra. For each of the stars, we created template spectra using the MARCS (Model Atmospheres in Radiative and Convective Scheme) stellar atmospheres and the Turbospectrum spectral synthesis code (Alvarez \& Plez 1998; Gustafsson et al. 2008; Plez 2008) with stellar parameters $T_{\text {eff }}, \log g$, and $[\mathrm{Fe} / \mathrm{H}]$ as given in Table 1. In FXCOR, we fitted a Gaussian to the crosscorrelation peak, from which the formal radial velocity uncertainties were determined following the method described in Tonry \& Davis (1979).

In stars that are so metal-poor as those in our sample, not many lines are present in the spectrum. Only a few features can be used to determine robust radial velocities, the main features being the calcium triplet (at 8498,8542 and $8662 \AA$ ), the $\mathrm{H} \alpha$ line $(6563 \AA)$, and the magnesium triplet $(5167,5173$ and $5184 \AA)$. Blueward of these features, the $\mathrm{S} / \mathrm{Ns}$ tend to be too low for good radial velocity measurements.

\subsubsection{CFHT sample}

For our CFHT sample, we used the magnesium triplet region to determine good radial velocities. We found that the $\mathrm{H} \alpha$ line is too broad for precise radial velocities, and when we used the calcium triplet, we found a slight offset $\left(\sim 0.8 \mathrm{~km} \mathrm{~s}^{-1}\right)$ for the standard stars with respect to the literature (see Fig. 1). This figure shows that although the formal uncertainties on the radial velocities derived from the magnesium triplet are larger than those from the calcium triplet, the velocities are more accurate. Additionally, for the CEMP-no stars that overlap with the $\mathrm{H} 16 \mathrm{a}$ sample, the radial velocities of the magnesium triplet agree better with the H16a velocities than those from the calcium triplet. Our supposition is that the difference between the radial velocities of the calcium and magnesium triplet is the result of a faulty wavelength calibration in the red part of the ESPaDOnS spectra. Therefore we decided to use the radial velocities of the magnesium triplet in our analysis for the CFHT spectra, with uncertainties as provided by FXCOR.

\subsubsection{SALT sample}

The SALT spectra have lower S/Ns than the CFHT spectra and were taken at lower resolution. For these observations we therefore used the spectra from the red and blue arms to obtain more precise radial velocities.

To correct for any instabilities in the instrument, we computed two radial velocity corrections. First of all, we computed a correction using the telluric lines in the red spectrum. Telluric lines have fixed wavelengths and can be used to correct for exposure-to-exposure differences in the instrument that may cause changes in the wavelength solution. We applied this 




Fig. 1. Radial velocities of standard stars observed with CFHT compared to their literature values. The expected zero-line is indicated.

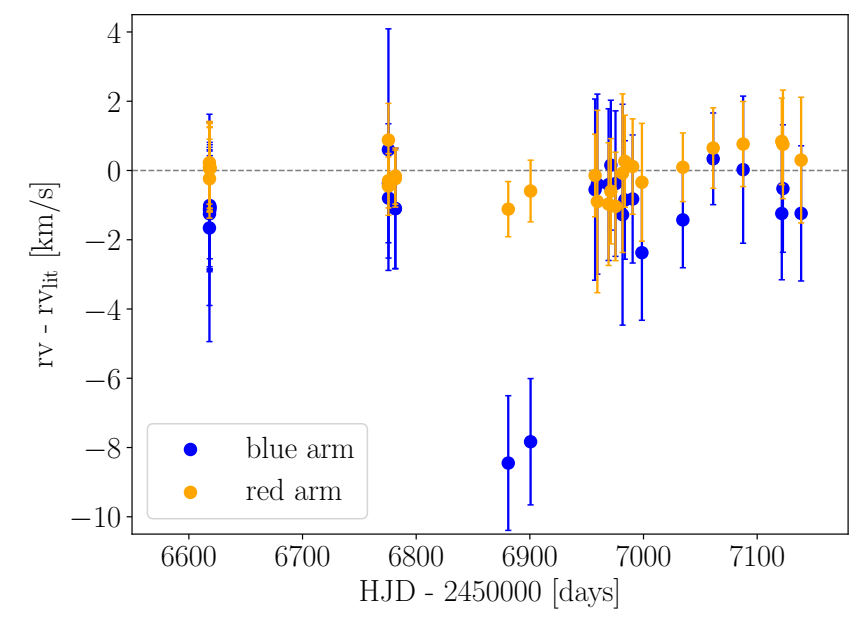

Fig. 2. Radial velocities of standard stars observed with SALT compared to their literature values. The expected zero-line is indicated.

correction to the blue and red spectrum of that observation, since the blue spectra show no telluric lines. For the observed radial velocity standard stars, we present the telluric-corrected radial velocities compared to the literature velocity in Fig. 2 . The red arm velocities agree well with the literature within the uncertainties of about $1 \mathrm{~km} \mathrm{~s}^{-1}$ for all standard stars throughout the full time-span, demonstrating that our radial velocity determination, when combined with the telluric correction, is robust and that the uncertainties are realistic.

The blue arm velocities for the standard stars show a small offset in most of the measurements. Furthermore, the blue-red difference around HJD-2 $450000=6900$ days (August 2014) is particularly large for the two observed standard stars. The history of maintenance operations on HRS shows that in August 2014, the instrument was plagued by several issues. The vacuum was lost a few times during the first weeks of August, and afterwards, the cameras needed to be heated up by more than 150 degrees to remove possible contaminants. Since this was done at least twice in this period, it is possible that in the process, one of the CCDs moved slightly. This could lead to a discrepancy between the two arms.

However, we can correct for the blue arm differences using the standard star observations. We applied a correction to the blue arm radial velocities for our science observations, which is the difference between the literature and measured radial velocity from the blue arm of the standard star(s) observed on the same night. Especially for the two science stars observed in August 2014, this drastically improves the consistency between the blue and red arm radial velocities. During one night, the blue arm correction may vary by up to $1 \mathrm{~km} \mathrm{~s}^{-1}$, and unfortunately, not every science observation always has its own radial velocity standard observation. Therefore, we inflated the FXCOR uncertainties on the blue arm radial velocities of the science observations by $1 \mathrm{~km} \mathrm{~s}^{-1}$.

The final radial velocities are computed as the weighted average of the (telluric and standard corrected) red and blue velocities, where the weights are the uncertainties as provided by FXCOR (with the blue uncertainties inflated). Final radial velocity uncertainties are estimated by the standard deviation of the two velocities derived from the blue and red arm.

For almost half of the spectra no radial velocity standard star was observed on the same night. For these stars we did not use the blue arm radial velocities, but accepted the radial velocities from the red arm with uncertainties as provided by FXCOR. This resulted in lower precision for these stars, but as illustrated in Fig. 2, we are confident that the red arm velocities are accurate. Additionally, for two nights (five spectra) no red spectrum was available for science or standard stars, and therefore also no telluric correction. We excluded these measurements from this work entirely. For the five stars with SALT data that overlap with the H16a sample, the derived radial velocities agree with those from $\mathrm{H} 16 \mathrm{a}$ within the uncertainties.

\subsection{CEMP compilation}

For an overview of known CEMP stars, we have used data from Yoon et al. (2016) as a basis. These authors have compiled a large number of CEMP stars from the literature. The sample contains 127 CEMP-no stars, 147 CEMP- $s$ stars, and 31 unclassified CEMP stars based on upper limits on their $[\mathrm{Ba} / \mathrm{Fe}]$. The carbon abundances in this compilation were corrected for evolutionary phase (following Placco et al. 2014), and throughout this paper, we use their corrected $[\mathrm{C} / \mathrm{Fe}]$ and $\mathrm{A}(\mathrm{C})$ values for the stars in our sample (Table 1) and when we refer to the compilation.

We added six stars to this compilation: the recently discovered hyper metal-poor star SDSS J0815+4729 from Aguado et al. (2018), which is extremely enhanced in carbon (with $[\mathrm{Fe} / \mathrm{H}]<-5.8$ and $[\mathrm{C} / \mathrm{Fe}]>+5.0$ ), the recently discovered CEMP-no binary star SDSS J1341+4741 from Bandyopadhyay et al. (2018), CS 22166-016 (Giridhar et al. 2001) and CS 22878-027 (Yong et al. 2013), which are two CEMP-no stars that were monitored in radial velocity by $\mathrm{H} 16 \mathrm{a}$ and us and which are missing in the compilation, and G6412 and G64-37, which were found to be CEMP-no stars by Placco et al. (2016a). For SDSS J0929+0238 we updated the $\log g$ (to the main-sequence solution), $[\mathrm{Fe} / \mathrm{H}],[\mathrm{C} / \mathrm{Fe}]$, $\mathrm{A}(\mathrm{C})$, upper limit for $[\mathrm{Ba} / \mathrm{Fe}]$, and binary status to the values in Caffau et al. (2016). We updated the $[\mathrm{Fe} / \mathrm{H}],[\mathrm{C} / \mathrm{Fe}]$, and A(C) of HE 2319-5228 to the values from Beers et al. (2017). HD 135148 is a known binary star (Carney et al. 2003), therefore we updated its binary status in the compilation. The star has a $[\mathrm{Ba} / \mathrm{Fe}]=+0.3$ (Simmerer et al. 2004) and is not clearly classified as CEMP-no or CEMP- $s$, therefore we did not consider this star in our analysis. For SMSS 0313-6708 we updated the $[\mathrm{Fe} / \mathrm{H}]$ upper limit to -6.5 (Nordlander et al. 2017) .

For HE 1201-1512 and SDSS J0140+2344, Yoon et al. (2016) only provided the subgiant solutions, even though 
Yong et al. (2013) provided both dwarf and subgiant solutions since the evolutionary status of this star was unknown at the time. With Gaia DR2 (Gaia Collaboration 2018), we can determine which solution is likely the best one. We compared the $\mathrm{BP}-\mathrm{RP}$ and absolute $\mathrm{G}$ magnitude (converted using the parallax) to a MIST isochrone ${ }^{4}$ (Dotter 2016; Choi et al. 2016) with $[\mathrm{Fe} / \mathrm{H}]=-4.0$ at an age of $12.5 \mathrm{Gyr}$. We find that both stars are more consistent with being dwarfs, therefore we accept the dwarf solutions.

\section{Results}

\subsection{Radial velocity database}

We present all derived radial velocities for the stars in our CFHT/SALT sample in Table A.1. We supplemented our radial velocities with values from the literature, to obtain a time span as long as possible and as many radial velocities for as many stars as possible. We compiled a list with all available radial velocity measurements from $\mathrm{S} 14, \mathrm{H} 16 \mathrm{a}$, and this work, and added five stars from the literature. The CEMP-no stars G77-61 (Dearborn et al. 1986), SDSS J0929+0238 (Caffau et al. 2016), and SDSS J1341+4741 (Bandyopadhyay et al. 2018) are known to vary in radial velocity. G77-61 and SDSS J0929+0238 only have upper limits for $[\mathrm{Ba} / \mathrm{Fe}]$, but are assumed to be CEMP-no stars because they are not on the high-carbon band. SDSS J0929+0238 has been discovered as a double-lined (possibly even triple-lined) spectroscopic binary, and it was monitored for radial velocity variations after. For this star, two or three radial velocities per spectrum are given for spectra that had multiple components (as in Caffau et al. 2016). The two stars G64-12 and G64-37 are CEMP-no stars that are constant in radial velocity (Latham et al. 2002). Additionally, we kindly received several unpublished radial velocity measurements from N. Christlieb for HE 0557-4840 and HE 0107-5240, which we have added to the compilation.

We supplemented our literature sample by deriving a radial velocity for other available spectra, using the same method as for the CFHT spectra. For HE 1201-1512, we derived the radial velocity from a FEROS spectrum that was taken during followup efforts from the Pristine survey (Starkenburg et al. 2017; Caffau et al. 2017). We also searched the ESO archive and found a UVES spectrum for SDSS J0140+2344 taken as part of the TOPoS survey (Bonifacio et al. 2018) and 34 UVES spectra over the course of one year for the most iron-poor star SMSS J03136708 , which was observed as part of the SkyMapper extremely metal-poor star survey (Keller et al. 2014; Bessell et al. 2015). No radial velocities for SMSS J0313-6708 have previously been published.

Our efforts result in a sample of 710 individual radial velocity measurements (including this work) for 45 CEMP-no stars. The stars are listed in Table B.1 with their radial velocity properties, stellar parameters, and carbon and barium abundances. The individual radial velocity measurements can be found in Table B.2.

\subsection{Radial velocity variation in the sample}

For each of the 45 CEMP-no stars in the compilation, we determined the $\chi^{2}$ of the radial velocity distribution,

$\chi^{2}=\sum_{i=1}^{n}\left(\frac{v_{i}-\bar{v}}{\sigma_{v_{i}}}\right)^{2}$,

\footnotetext{
4 http://waps.cfa.harvard.edu/MIST/interp_isos.html
}

and used it to compute the probability that the radial velocity is constant, the $\mathrm{p}$-value $P\left(\chi^{2}\right)$. Before computing the $\chi^{2}$, we quadratically added to the radial velocity uncertainties from $\mathrm{H} 16 \mathrm{a}$ a floor uncertainty of $0.1 \mathrm{~km} \mathrm{~s}^{-1}$ to account for external uncertainty sources, as H16 did when computing their $\chi^{2}$. The final probability for each of the stars is presented in the fifth column of Table B.1. Carney et al. (2003) found that all binary stars in their sample have $P\left(\chi^{2}\right)<10^{-6}$, which is what we take as our binary candidate selection criterion.

Among the stars with $P\left(\chi^{2}\right)<10^{-6}$, we find the six known binary systems from the literature: CS 22957-027, HE 02191739, HE 1150-0428, and HE 1506-0113, which are the four binaries discussed in S14 and H16a, and additionally G77-61 and SDSS J1341+4741 (see Sect. 3.1). SDSS J0929+0238 is also binary, but is not included in this analysis since it is a spectroscopic double-lined system.

\subsubsection{New binary candidates}

There are five additional stars with $P\left(\chi^{2}\right)<10^{-6}$, which are good binary candidates. Three of them, HE0107-5240, HE 2139-5432 and SDSS J1422+0031, are in our southern hemisphere SALT sample. The fourth star, SDSS J0140+2344, is in our CFHT sample and is one of the few stars in that sample that has not been monitored by H16a. Finally, SDSS J1313-0019 has not been observed by us, but comes from the literature.

We present the individual radial velocity measurements for the three stars with $>10 \mathrm{~km} \mathrm{~s}^{-1}$ variation that include measurements from this work in Fig. 3. SDSS J1422+0031 and SDSS J0140+2344 have been mentioned before as possible interesting candidates in S14.

The fourth star that includes measurements from this work, HE 0107-5240, is presented in Fig. 4. This star was long thought to be non-variable in radial velocity, but when we included our new measurements, this star appears to be varying on a long timescale ( $>10$ years). Our supposition is that it is part of a (wide) binary system.

The final star, SDSS J1313-0019, has three radial velocity measurements in the literature, of which two come from low-resolution spectra $\left(268 \pm 4 \mathrm{~km} \mathrm{~s}^{-1}\right.$ and $242 \pm 4 \mathrm{~km} \mathrm{~s}^{-1}$ from SEGUE and BOSS, respectively, Allende Prieto et al. 2015) and one from a high-resolution spectrum $\left(274.6 \mathrm{~km} \mathrm{~s}^{-1}\right.$, no uncertainty given, Frebel et al. 2015). Both teams of authors have suggested that this star might be in a binary system, but more measurements are needed to confirm or refute this. We do not treat this star as a binary system in this work because it has only one measurement from high-resolution spectroscopy.

\subsubsection{Other stars with low $P\left(\chi^{2}\right)$}

Two additional stars had $P\left(\chi^{2}\right)<10^{-6}$. The first, HE 1410+0213, was observed extensively by $\mathrm{H} 16 \mathrm{a}$, and after much analysis, they concluded that the star is most likely single. They assumed that the velocity variation comes from low-amplitude pulsations in the star and suggested adding a velocity jitter of $0.15 \mathrm{~km} \mathrm{~s}^{-1}$. When we add such a jitter, $P\left(\chi^{2}\right)$ is increased (as presented in Table B.1).

The second star is the most iron-poor star of the compilation, SMSS 0313-6708. Its 34 radial velocities (which we derived from archive UVES spectra) measured over the course of one year have a dispersion of $0.4 \mathrm{~km} \mathrm{~s}^{-1}$, with two measurements that have a difference of $2.3 \mathrm{~km} \mathrm{~s}^{-1}$ (see Fig. C.1). We were not able to fit an orbit through all points using the method in Sect. 3.3 


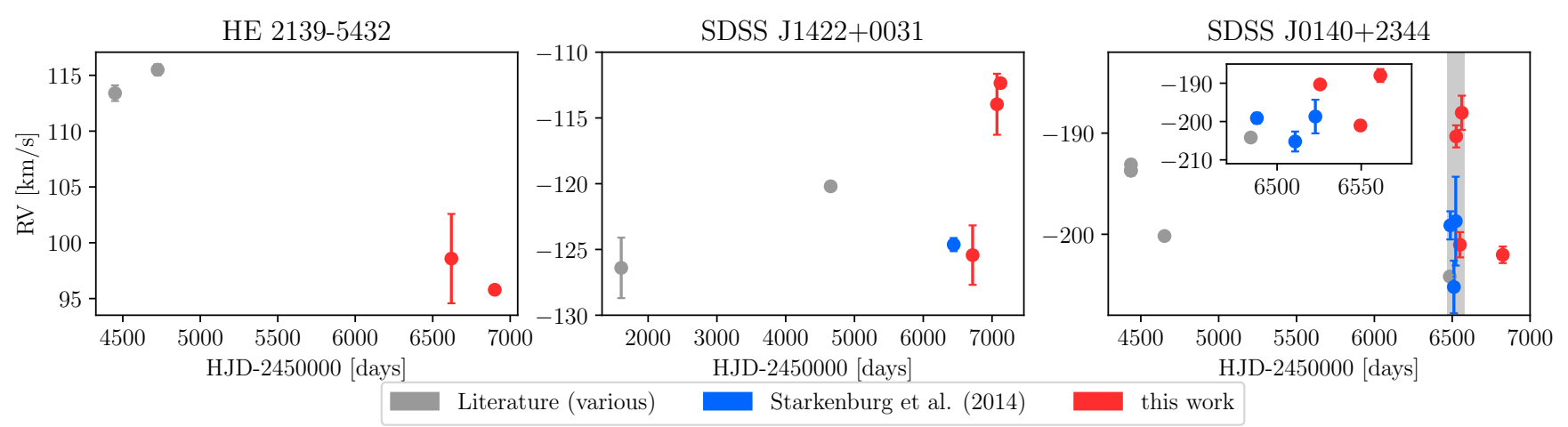

Fig. 3. Radial velocities for the three stars in our sample that strongly vary in radial velocity $\left(>10 \mathrm{~km} \mathrm{~s}^{-1}\right)$ and were not previously identified as binaries. For SDSS J0140+2344, a zoom-in of the shaded region is shown.

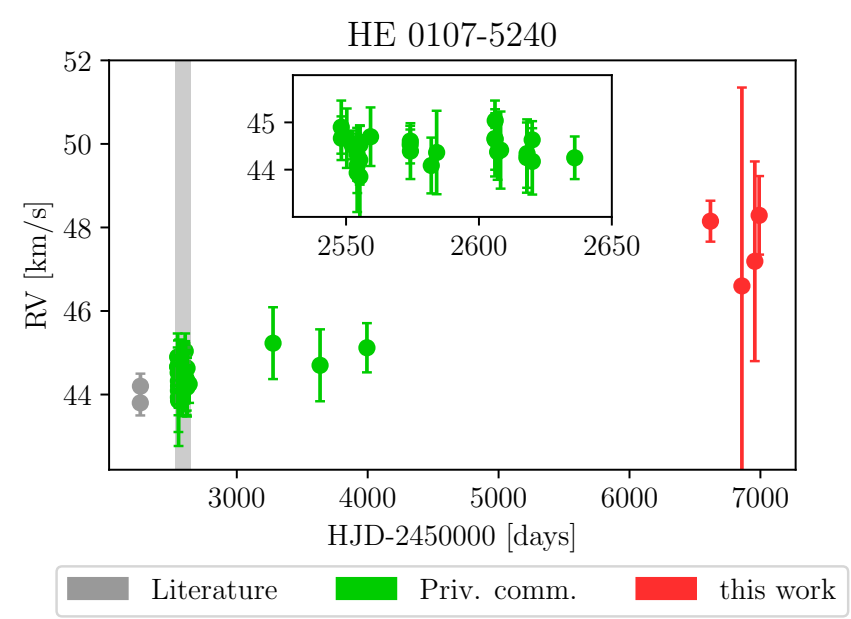

Fig. 4. Radial velocities for HE0107-5240, including those unpublished values that we received through N. Christlieb (priv. comm.). A zoom-in of the shaded region is shown.

and, excluding the two outliers, the other measurements seem compatible with a constant radial velocity. We therefore conclude that it is likely that the radial velocity uncertainties are underestimated. The ESO archive lists no radial velocity standard stars that are observed on the same nights as the two outliers, therefore we cannot correct for any systematics. Instead, we estimate an uncertainty floor by assuming that the star does not vary in radial velocity. We quadratically added a constant to each of the the uncertainties until a final reduced $\chi^{2}$ of 1 was reached. This leads to an uncertainty floor of $0.35 \mathrm{~km} \mathrm{~s}^{-1}$, which we have added to each measurement. We provide these inflated uncertainties for SMSS 0313-6708 in Table B.2. After this correction, this star no longer has $P\left(\chi^{2}\right)<10^{-6}$ (the updated value is presented in Table B.1). In this work we treat SMSS 0313 6708 as single, although it could still vary in radial velocity on a timescale longer than one year.

\subsubsection{Final notes on radial velocity variations}

Radial velocity variations are not only caused by binarity, they can also be caused by inhomogeneities on the surfaces of stars or stellar pulsations, for example. Carney et al. (2008) found that the velocity "jitter" that is due to inhomogeneities mainly affects evolved stars with $M_{V} \leq-1.4(\log g \lesssim 1.0)$. None of our four new binary candidates are in this regime, nor do they lie in parts of the HR diagram that are known for stellar pulsations. We therefore conclude that their radial velocity variations are due to binarity.

For all remaining stars in our CFHT/SALT sample we present the individual radial velocity measurements in Fig. C.2. Our measurements agree with previous measurements when there is overlap.

\subsection{Orbit properties of the new binaries}

We applied the code THE JoKER (Price-Whelan \& Hogg 2017; Price-Whelan et al. 2017) to the radial velocity data of our four newly discovered binary systems: HE 0107-5240, HE 21395432, SDSS J0140+2344, and SDSS J1422+0031. ThE JoKer is a Monte Carlo sampler for orbital parameters of binary systems that can also be applied to sparse and/or low-quality radial velocity data. It produces a posterior sampling of the period, eccentricity, pericentre phase and argument, velocity semi-amplitude, and the barycentre velocity. For all four of our stars, the eccentricity and pericentre phase and argument were not well constrained in the analysis. The resulting corner plots (Foreman-Mackey 2016) for the periods and semi-amplitudes provide insight, however, and can be found in Fig. 5. We took $10^{6}$ samples per star, except for SDSS J0140+2344, where we took $10^{8}$ because the orbit is relatively well determined with the available radial velocity measurements, so that few samples are accepted.

The analysis with THE JOKER for HE 0107-5240 results in a period distribution that peaks between 10000 and 30000 days (27-82 years) and a semi-amplitude of the order of $2-5 \mathrm{~km} \mathrm{~s}^{-1}$. HE 2139-5432 has sparse radial velocity data that allow for many possible short-period orbits up to 300 days, or longer period orbits of $\sim 4000$ days, both with semi-amplitudes of $\sim 11 \mathrm{~km} \mathrm{~s}^{-1}$. For SDSS J0140+2344 we find a narrow peak of the period distribution at 32 days, and a semi-amplitude of $7.5 \mathrm{~km} \mathrm{~s}^{-1}$. Finally, for SDSS J1422+0031 we find multiple peaks, the most pronounced one producing a period of $\sim 1600$ days and a semi-amplitude of $\sim 8 \mathrm{~km} \mathrm{~s}^{-1}$. Clearly, for at least three of these stars, more radial velocity measurements are needed to determine better orbital solutions.

\section{Properties of the CEMP-no radial velocity sample}

By combining our four new CEMP-no binaries with the four known binaries discussed in S14 and H16a (CS 22957027, HE 0219-1739, HE 1150-0428, and HE 1506-0113) and the three literature stars G77-61 (Dearborn et al. 1986), SDSS J0929+0238 (Caffau et al. 2016) and SDSS J1341+4741 (Bandyopadhyay et al. 2018), we have a sample of eleven 

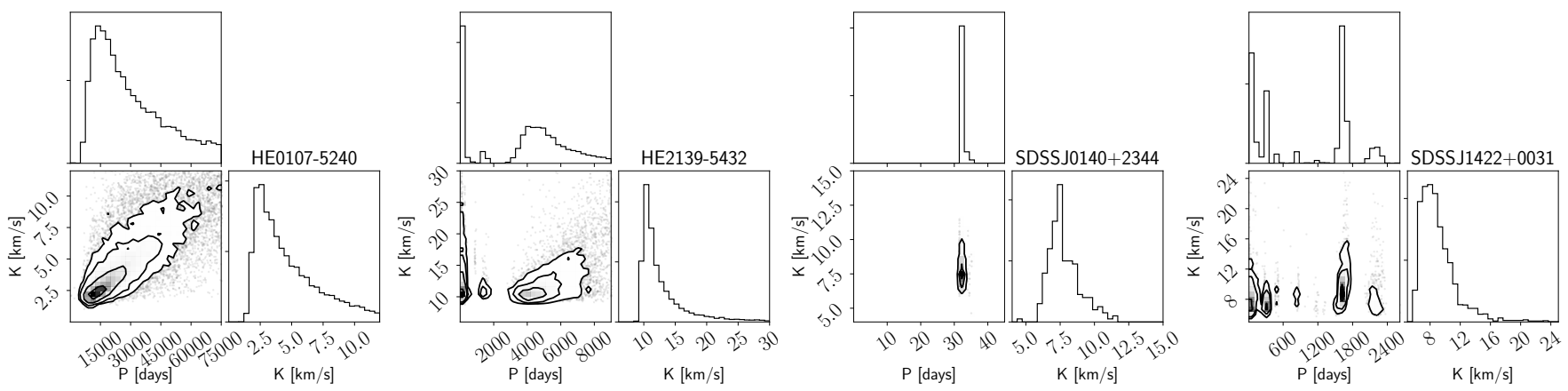

Fig. 5. Orbits solutions from the JoKer for the period $P$ and the semi-amplitude $K$ for the new binaries. The ranges on the x-and y-axes have been truncated for clarity, but at least the two largest peaks of the distribution are always shown.

CEMP-no binary stars. To obtain to a binary fraction, the number of single stars needs to be determined as well. Long monitoring time-scales and high radial velocity precision are needed to rule out the binarity of a star. If the timescale is too short or the uncertainties are too large, a long-period low-amplitude signal might be hiding in the data. For all practical purposes, however, we assumed that the monitored stars that do not show indications of radial velocity variations are single. We excluded from the single-star sample those stars with fewer than five radial velocity measurements, because the radial velocity precision and/or temporal coverage of those measurements is not sufficient to claim that a star is single based on so few data points. Combining the single and binary stars results in a binary fraction of $32_{-9}^{+10 \%}$ (11 out of 34) for the whole sample of radial velocity monitored CEMP-no stars, using binomial statistics to derive the $\pm 1 \sigma$ uncertainties. This binary fraction is larger than $17_{-8}^{+11 \%}$ (4 out of 24) as found by H16a for CEMP-no stars, but agrees within $1 \sigma$. The discrepancy of our result with the binary fraction of $16_{-4}^{+5 \%}$ (14 out of 85 ) for carbon-normal metal-poor $(-3.0 \lesssim[\mathrm{Fe} / \mathrm{H}] \lesssim-1.4)$ giants by Carney et al. (2003) is slightly larger than $1 \sigma$. However, both of these are not statistically significant differences.

The sample of H16a appears to be more homogeneously monitored than the complete combined sample in this work, because we and others in the literature may have preferentially monitored stars that already showed some indication of radial velocity variation. The binary fraction in this work might not necessarily be representative for the whole population.

All these binary fractions (including those from H16a and Carney et al. 2003) are more correctly thought of as lower limits, given that long-period binaries or binaries at high inclinations may still be present among the assumed single stars. It is much easier to confirm the binarity of a star than to rule out its binarity, since fewer measurements are needed to find that a star is variable in radial velocity, especially if the amplitude of the variation is large.

However, the collection of binary stars we now have is interesting to study in itself. In the following subsections we discuss some of the properties of the CEMP-no binary population.

\subsection{Orbit characteristics}

How do the orbit properties of CEMP-no stars compare to those of CEMP- $s$ stars and other metal-poor binary systems? We compare the periods and semi-amplitudes in Fig. 6. The uncertainties for the binaries from this work are taken from the $1 \sigma$ contours of the most significant peaks from the probability distributions in Fig. 5. There is no indication that the CEMP-no binaries are

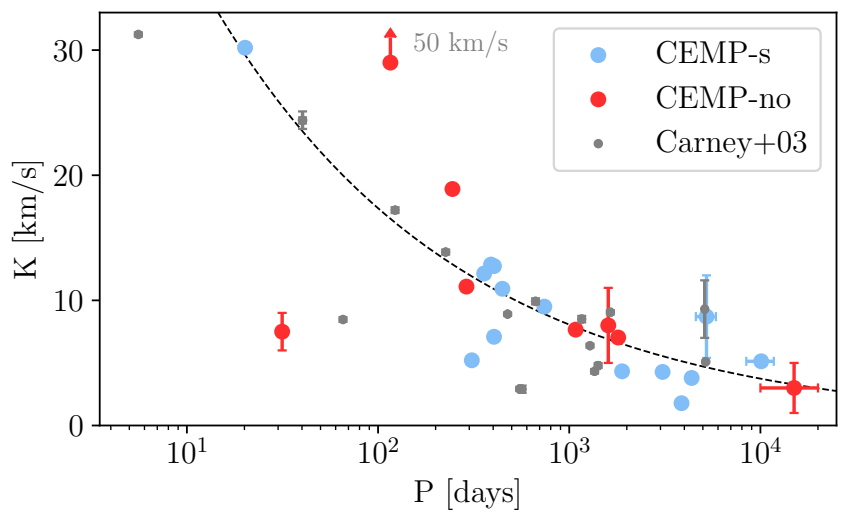

Fig. 6. Periods and semi-amplitudes of carbon-normal metal-poor binaries from Carney et al. (2003), CEMP-s binaries from Hansen et al. (2016a; excluding their CEMP- $r / s$ stars), and CEMP-no binaries (compilation from this work). HE 1506-0113 (see Fig. C.2) and SDSS J0929+0238 are not shown in this figure since they do not have any derived orbits, HE 2139-5432 is not shown because it either has $P<300$ or $P \approx 4000$ days (with $K \approx 11 \mathrm{~km} \mathrm{~s}^{-1}$ ). The line indicates what would be expected of Keplerian orbits of a $0.8 M_{\odot}$ star with a $0.5 M_{\odot}$ companion, for an eccentricity of 0.3 (typical for the stars in the Carney et al. 2003 sample) under an inclination of $60^{\circ}$.

of a different distribution than the CEMP- $s$ binaries or the stars from Carney et al. (2003). The typical companion mass for stars in the latter sample is $0.5 M_{\odot}$ with eccentricities of $\sim 0.3$. Only three of the CEMP-no binaries have known eccentricities, H16a claimed that their distribution is not different from normal metalpoor stars.

One odd star is SDSS J0140+2344 with its short period of 31.5 days and a relatively low semi-amplitude of $7.5 \mathrm{~km} \mathrm{~s}^{-1}$. This system may be observed relatively face-on. Alternatively, it may have a very low-mass companion. Schlaufman et al. (2018) discovered an ultra metal-poor binary system with a very similar period and semi-amplitude and claim that it has a $0.14 M_{\odot}$ companion, making the companion the lowest mass ultra metal-poor star known. Another odd star is SDSS J1341+4741, which has a reported period of 116 days and a semi-amplitude of $50 \mathrm{~km} \mathrm{~s}^{-1}$ (Bandyopadhyay et al. 2018). If this is confirmed with more radial velocity measurements, this star would have to have a more massive companion than the other stars or be on a highly eccentric orbit.

H16a were unable to derive an orbital solution for HE 15060113, despite the large number of radial velocity measurements and its clear variability. This star seems to vary on a short timescale ( $\sim 20$ days) in the data from Norris et al. (2013b), which have been reanalysed by H16a, and a longer timescale 

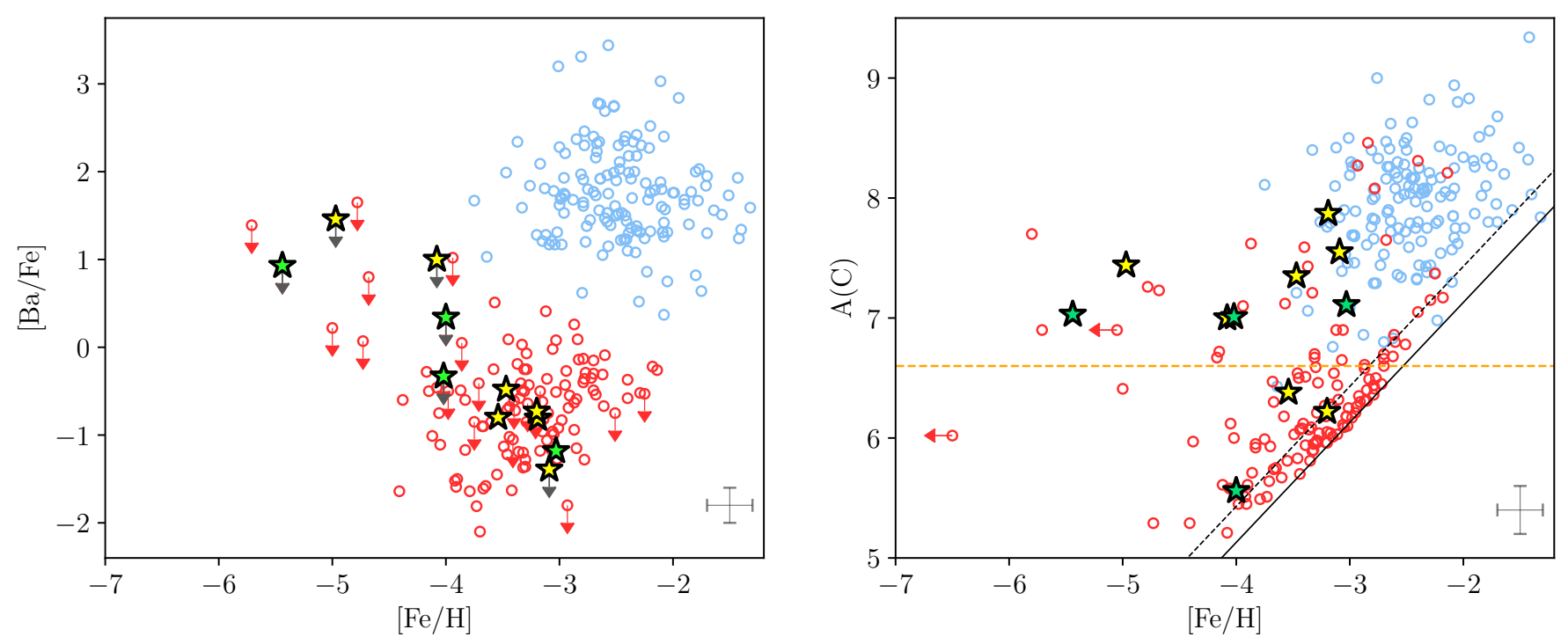

Fig. 7. Left panel: $[\mathrm{Ba} / \mathrm{Fe}]$ as a function of $[\mathrm{Fe} / \mathrm{H}]$ for the compilation of CEMP stars from Yoon et al. (2016) down to $[\mathrm{Fe} / \mathrm{H}]=-6$, where $\mathrm{CEMP}-s$ stars are shown in blue and the CEMP-no stars in red. The known and new CEMP-no binaries are indicated as yellow and green stars, respectively. Right panel: same, but for $\mathrm{A}(\mathrm{C})$ on the $\mathrm{y}$-axis. The solid and dotted black lines indicate the $[\mathrm{C} / \mathrm{Fe}]>+0.7$ and $[\mathrm{C} / \mathrm{Fe}]>+1.0 \mathrm{CEMP}$ criteria, respectively. The orange dashed line indicates the separation of the low- and the intermediate-/high-carbon band. For both panels, typical error bars on the abundances are shown in the lower right corner.

( 1000 days) on the basis of data from S14 and H16a, see Fig. C.2. Our new radial velocities for this star fill the gap between the measurements of S14 and H16a, but do not help to clarify its orbit.

\subsection{Enhancement in s-process elements}

Enhancement in the $s$-process element barium is usually a sign of mass transfer from an AGB companion. We present $[\mathrm{Ba} / \mathrm{Fe}]$ as a function of $[\mathrm{Fe} / \mathrm{H}]$ in the left panel of Fig. 7 for our binary stars on top of the CEMP compilation of Yoon et al. (2016), where the CEMP-no stars are shown in red and the CEMP- $s$ stars in blue. The CEMP-no binaries from the literature (from S14, H16a, Dearborn et al. 1986; Caffau et al. 2016; Bandyopadhyay et al. 2018) are shown as yellow stars, and the new binaries uncovered in this work are plotted as green stars. CEMP star classes are defined mainly by the barium abundance, therefore the CEMP$s$ stars (blue points) and the CEMP-no stars (red points) are almost perfectly separated in this diagram. Four binaries have upper limits on $[\mathrm{Ba} / \mathrm{Fe}]$ that are above zero. HE 0107-5240 and SDSS J0140+2344 were discussed in Sect. 2.1, and taking the revised CEMP-no definition of Matsuno et al. (2017), SDSS J0929+0238 is also classified as CEMP-no. Even though it does not satisfy the revised definition, the dwarf G77-61 $([\mathrm{Ba} / \mathrm{Fe}]<+1.0)$ is likely a CEMP-no star as well, assuming that all ultra metal-poor low-carbon band stars are. Alternatively, it could be the first ultra metal-poor $([\mathrm{Fe} / \mathrm{H}] \leq-4.0)$ CEMP$s$ star. However, since no ultra metal-poor CEMP-s stars are known to date, we assume that it belongs to the CEMP-no class.

The binaries seem to be part of the normal CEMP-no distribution in the left panel of Fig. 7. The low $[\mathrm{Ba} / \mathrm{Fe}]$ values (or upper limits) of most the binary stars are consistent with them having had no "classical" binary interaction with an AGB star in which $s$-process elements were transferred together with carbon.

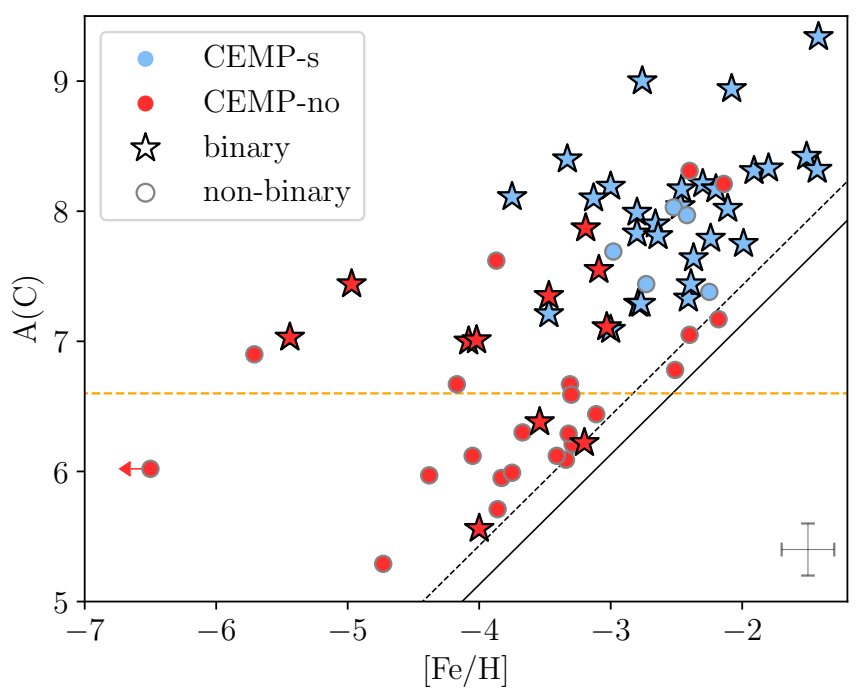

Fig. 8. $\mathrm{A}(\mathrm{C})$ as a function of $[\mathrm{Fe} / \mathrm{H}]$ for $\mathrm{CEMP}$ stars, where again CEMP-no stars are shown in red and CEMP- $s$ stars in blue. Binary stars are indicated by a star. The orange dashed line is the same as in the right panel of Fig. 7.

\subsection{Absolute carbon abundance}

As discussed in the introduction, in general CEMP- $s$ and CEMPno stars have different absolute carbon abundances. This can be seen in the right panel of Fig. 7 for the compilation of CEMP stars. Yoon et al. (2016) suggested that there may be three different groups of CEMP stars based on their $\mathrm{A}(\mathrm{C})$ and $[\mathrm{Fe} / \mathrm{H}]$. First, there are the Group I stars that cover the region of the CEMP- $s$ stars at large $\mathrm{A}(\mathrm{C})$ and higher $[\mathrm{Fe} / \mathrm{H}]$. Then there are two different groups of CEMP-no stars, the Group II stars, that in general have lower $\mathrm{A}(\mathrm{C})$ and exhibit a clear dependence of $\mathrm{A}(\mathrm{C})$ on $[\mathrm{Fe} / \mathrm{H}]$, and the Group III stars that seem to centre on a higher $\mathrm{A}(\mathrm{C})$ without a clear dependence on $[\mathrm{Fe} / \mathrm{H}]$. 
One might expect that like the CEMP-s stars, most highcarbon band CEMP-no stars would be in binary systems. However, H16a did not find a strong correlation between the binary status of CEMP-no stars and their location on the $\mathrm{A}(\mathrm{C})$ versus $[\mathrm{Fe} / \mathrm{H}]$ plane (although that is difficult to say with such a small sample). In the right panel of Fig. 7 we highlight the 11 currently known CEMP-no binaries on the $\mathrm{A}(\mathrm{C})$ versus $[\mathrm{Fe} / \mathrm{H}]$ plane. It appears that most of the CEMP-no binaries have relatively high $\mathrm{A}(\mathrm{C})$ values in between the high- and low-carbon bands of Spite et al. (2013), which seems to correspond roughly to the region of the Group III stars of Yoon et al. (2016; although four of the binary stars are classified as Group I stars).

This does not seem to be a selection effect of only monitoring stars with the highest carbon enhancement, which can be seen from Fig. 8. There we present the same $\mathrm{A}(\mathrm{C})$ versus $[\mathrm{Fe} / \mathrm{H}]$ plane as in Fig. 7, but now only for stars that have sufficient radial velocity data to determine with some confidence whether they are in a binary system or not. Typically, the stars that we assume to be single have been observed as thoroughly as the other stars, but we cannot fully exclude the possibility that they are in a binary system with a long period or low amplitude. For the CEMP- $s$ stars, we used the binarity information as documented in Yoon et al. (2016).

In Fig. 8 the fraction of CEMP-no stars that are in binary systems seems higher for stars on the intermediate- or high-carbon band compared to the stars on the low-carbon band. Splitting our sample of well-monitored stars in half based on $\mathrm{A}(\mathrm{C})$, as illustrated by the orange dashed line in Fig. 8, we find that for the CEMP-no stars with $\mathrm{A}(\mathrm{C})>6.6$ the binary fraction is $47_{-14}^{+15} \%(8$ out of 17), and for $A(C) \leq 6.6$ it is $18_{-9}^{+14} \%$ (3 out of 17). The difference between these two fractions is $1 \sigma$.

If we conservatively assume that except for the known binary CEMP-no stars, all the known CEMP-no stars with $\mathrm{A}(\mathrm{C})>6.6$ are single stars (even if they do not have any radial velocity information), we find a binary fraction of $18_{-6}^{+8} \%$ (8 out of 44 ). This conservative lower limit for the binary fraction of high-carbon CEMP-no stars is independent of the selection for radial velocity monitoring or the quality of the determination of single stars.

The periods for CEMP-no binary stars with $\mathrm{A}(\mathrm{C})>6.6$ and derived orbits are similar to the periods for CEMP-s binary stars, which typically are of the order of a few 100 to a few 1000 days (see Fig. 6). The three CEMP-no binaries with $\mathrm{A}(\mathrm{C})<6.6$ are HE 1506-0113, SDSS J0140+2344 and SDSS J1341+4741. It is curious that each of these three stars was described in Sect. 4.1 because they have no or odd orbital solutions.

We end this section with a note of caution: the (absolute) carbon abundances need to be interpreted with caution, since most of the measurements were not made using non-local thermodynamic equilibrium (non-LTE) and/or 3D models, and such corrections may be especially important for carbon when comparing stars of different metallicities and evolutionary stages.

\subsection{Hertzsprung-Russell diagram}

We highlight the location of the CEMP stars on the (spectroscopic) Hertzsprung-Russell diagram in Fig. 9, where again we only show the stars that have sufficient radial velocity information available. Almost all the stars in the compilation are giant stars, since these are intrinsically brighter and easier to analyse. Additionally, CEMP stars with lower effective temperatures are easier to recognise from the spectra because the $\mathrm{CH}$ features are more distinct. Three CEMP-no binary stars lie on the main sequence (or close to the turn-off), G77-61 with $T_{\text {eff }}=4000 \mathrm{~K}$,

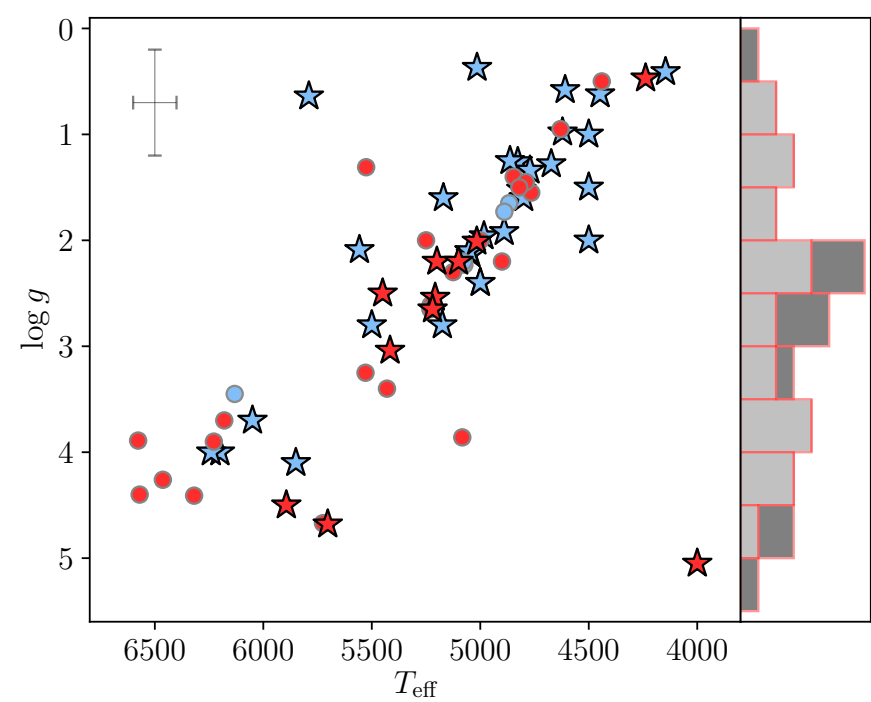

Fig. 9. Hertzsprung-Russell diagram for all CEMP-no and CEMP- $s$ stars, with the same symbols and colour-coding as in Fig. 8. Because the narrow giant branch is very crowded, we additionally show for the CEMP-no stars the stacked histogram for $\log g$ on the side, where dark grey are binary and light grey are single stars. Typical error bars are indicated in the top left corner.

SDSS J0140+2344 with $T_{\text {eff }}=5703$ and $\log g=4.7$, and SDSS J0929+0238 with $T_{\text {eff }}=5894 \mathrm{~K}$ and $\log g=4.5$. Another possibility is that SDSS J0929+0238 is a sub-giant branch star with $\log g=3.7$, but it has been argued by Caffau et al. (2016) that the main-sequence solution is more likely (unfortunately, the Gaia DR2 parallax for this star is uncertain).

One of the CEMP-no binary stars, HE 0219-1739, is at the tip of the giant branch. The stellar parameters for this star are somewhat uncertain, however. T. Hansen found $T_{\text {eff }}=4600$ and $\log g=2.3$ with stacked high-resolution radial velocity monitoring spectra (priv. comm.). Unfortunately, the Gaia parallax for this star is negative and does not help to constrain the $\log g$. The question arises whether this star is a real CEMP star, or if it might be an intrinsic (pulsating) carbon star. The welldetermined radial velocity period of this star is 1800 days, which is longer than typical pulsation periods of long-period variable (LPV) stars. This star is also indicated as variable in its photometry by PanSTARRS (Hernitschek et al. 2016), but the given variability timescale ( 0.13 days) is too short and the magnitude amplitude (0.034 mag in $r$ ) is too small to represent LPV pulsations. We conclude that this star is most likely a bona fide CEMP star in a binary system.

The apparent over-density of CEMP-no binary stars between $\log g=2-3$ as illustrated in a histogram in the right panel of Fig. 9 seems curious, even though we have monitored stars along the entire giant branch and turn-off. In comparison, the general sample of CEMP-no stars (from the Yoon et al. 2016 compilation) does not show an over-density between $\log g=2-3$. Larger samples are needed to put this tentative result on firmer footing.

We note that compared to CEMP-no stars, a larger number of monitored CEMP-s stars appear to lie on the upper part of the red giant branch. It is unlikely that this is a brightness selectioneffect, since the stars in the samples of H16a and Hansen et al. (2016a) for CEMP-no and CEMP- $s$ stars cover similar ranges in $V$ magnitudes (and they make up most of the stars in this diagram). Other unknown selection effects might play a role, however. 


\section{Discussion}

\subsection{CEMP-no stars and binary mass transfer}

Each of the CEMP-no binary stars must have a companion that causes its radial velocity variations. Since none of the stars except SDSS J0929+0238 seem to be double-lined spectroscopic binaries and no visual counterpart is seen in photometry of any of the stars, the companion must be a fainter star. For the dwarf star G77-61 it has been argued that the companion is most likely a white dwarf (Dearborn et al. 1986). For the giants, the companion can be expected to be a main-sequence star or a white dwarf (which has gone through the AGB phase in the past). Would it therefore not be likely that if some of the CEMP-no stars have white dwarf companions, they might have been polluted by mass transfer from an AGB companion at some point in their life?

For CEMP- $s$ stars, mass transfer from an AGB star is the main formation scenario, which has been confirmed by the number of them that is in a binary system $(82 \pm 10 \%$, Hansen et al. 2016a). Different types of mass-transfer are effective at different initial separations of the stars in the binary system, where Rochelobe overflow can occur in the closest systems, wind-assisted Roche-lobe overflow in systems of intermediate separation, and wind transfer by itself in wider systems. As shown in Fig. 6, typical periods and radial velocity amplitudes for binary CEMP$s$ and CEMP-no stars are similar. How many of the CEMP-no stars would have experienced binary interaction?

A simple estimate of the general fraction of metal-poor stars that interact with a binary companion can be made using the CEMP- $s$ stars, assuming that they all became CEMP- $s$ by masstransfer from a former AGB star. Of all stars with $[\mathrm{Fe} / \mathrm{H}]<-2.0$, $13 \%$ are CEMP-s stars (Placco et al. 2014) and therefore in a formerly interacting binary system. There is no reason (yet) to assume that at lower metallicity, binary stars suddenly start to interact less. By re-examining different surveys, Moe et al (2018) have found that the close binary fraction $\left(P<10^{4}\right.$ days $)$ of low-mass stars $\left(0.6-1.5 M_{\odot}\right)$ increases with decreasing metallicity. Therefore, in the regime of the CEMP-no stars, which are at lower metallicity than the CEMP- $s$ stars, an even larger fraction of all stars might be expected to have had interaction with a companion, simply because the binary fraction is higher. We see almost no CEMP- $s$ stars with $[\mathrm{Fe} / \mathrm{H}]<-3.0$, however, and none at all with $[\mathrm{Fe} / \mathrm{H}]<-4.0$. What do extremely metal-poor stars look like that underwent mass-transfer from a former extremely metal-poor AGB companion?

Our CEMP-no binaries are not enhanced in $s$-process elements (specifically barium), which has usually been taken as a sign that they have not had any interaction with a binary companion. However, much is unknown about ultra metal-poor AGB stars (see the recent review by Gil-Pons et al. 2018). Models of AGB transfer among metal-poor stars have focussed on CEMP- $s$ stars (as in Abate et al. 2015), which are mostly found at higher metallicities than the CEMP-no stars (see Fig. 7). The most metal-poor AGB yields available with $s$-process elements only extend to $[\mathrm{Fe} / \mathrm{H}]=-2.3$ (Lugaro et al. 2012). Extremely or even more metal-poor AGB stars might produce fewer $s$-process elements, for example in the (non-rotating) models of Suda et al. (2004), Lau et al. (2007), and Cruz et al. (2013). Additionally, rotation can strongly affect the $s$-process element production, for example in intermediate-mass spinstars (Meynet et al. 2010). Furthermore, the mass of the AGB star is important. It is expected that intermediate-mass AGB stars produce fewer $s$-process elements than AGB stars of lower mass, which especially affects $s$-process elements beyond the first $s$-process peak (Karakas \& Lattanzio 2014). If these are the polluting companion stars, no barium excess should be expected. Finally, Busso et al. (1999) suggested that in very metal-poor AGB stars, the $s$-process mainly produces third-peak $s$-process elements, particularly lead, instead of first or second $s$-process peak elements (like barium). However, lead is extremely hard to measure in carbon-enhanced metal-poor stars.

To summarise, AGB stars have ways to produce less barium and/or other $s$-process elements than usual. It is unclear what abundance patterns exactly are expected in extremely metal-poor AGB stars, and therefore what their companions should look like that have received mass-transfer from such a star.

\subsection{High fraction of binaries among intermediate- and high-carbon band CEMP-no stars}

\subsubsection{Scenario with binary interaction}

In a scenario in which a carbon-normal metal-poor star or an existing low-carbon band CEMP-no star in a binary system is (further) enriched in carbon by mass-transfer from such an AGB star, this would bring them up to the intermediate- or high$\mathrm{A}(\mathrm{C})$ band without enhancing their barium. Some or all of the carbon for these CEMP-no stars can be intrinsic, but likewise, some or all of it can come from mass transfer from a former AGB companion. If a number of CEMP-no stars were additionally enhanced in carbon over their lifetime and some were only intrinsically carbon-enhanced, this might be a possible explanation for the potential discrepancy in binary fraction between the higher $\mathrm{A}(\mathrm{C})$ and the low-A $(\mathrm{C})$ populations of CEMP-no stars.

It is not likely that mass-transfer from a companion has occurred in all the intermediate- or high-A(C) CEMP-no binary stars. For example, SDSS J0929+0238 with $\mathrm{A}(\mathrm{C})=7.44$ is a double-lined (possibly even triple-lined) spectroscopic binary of two main-sequence stars with similar temperatures (Caffau et al. 2016). If the carbon comes from mass-transfer from an AGB companion, these stars were born in a hierarchical triple system with the third star more massive and finally polluting the other two. This scenario decreases in likelihood if it were confirmed that SDSS J0929+0238 is a triple-lined system, which means that it consists of three main-sequence CEMP-no stars. In this case, the system would have had to been born as a quadruple system with one star being more massive and having evolved through the AGB phase. A better scenario probably is that these stars were born with an intrinsically high $\mathrm{A}(\mathrm{C})$, with the carbon formed in the previous generation of stars (as in the spinstar and/or faint supernova models).

Interestingly, five CEMP-s stars do not show any radial velocity variation. The binary fraction of CEMP- $s$ stars determined in Hansen et al. (2016a), $82 \pm 10 \%$, is not necessarily consistent with $100 \%$ binarity. The authors claimed that even with the uncertainties on the inclination, it is unlikely that all the apparently single stars are actually in binary systems. We note that in Fig. 8 the single CEMP- $s$ stars seem to be preferentially located on the lower side of the $A(C)$ distribution of the CEMP-s stars. Choplin et al. (2017) modelled the abundances of the single CEMP- $s$ stars with massive spinstar models, and succeed for three out of the four modelled stars. Spinstar models are also employed to explain the abundances of CEMP-no stars (e.g. Meynet et al. 2010). Potentially, single intermediateor high-carbon band CEMP-no stars (or binaries that have not had any interaction) and single CEMP- $s$ stars are the product of a similar type of progenitor.

A takeaway from this section is that a combination of one or more of the "classical" scenarios for the formation of CEMPno stars and binary interaction complicates the interpretation 
of the abundance patterns of these stars. CEMP-no stars are usually thought to be direct probes of nucleosynthesis in the first stars and supernovae, but they might also be probes of extremely metal-poor AGB stars.

\subsubsection{Scenario without binary interaction}

An alternative (or supplement) to the mass-transfer scenario is a scenario where binary stars form more easily in a carbon-enhanced environment. For example, Chiaki et al. (2017) have shown that for $[\mathrm{C} / \mathrm{Fe}]<+2.30$, silicate dust grains dominate the cooling during star formation of extremely metalpoor stars, while for $[\mathrm{C} / \mathrm{Fe}]>+2.30$, carbon grains dominate. It is not known how this would affect the binary fraction, but there might a difference between these environments of different dust cooling. Unfortunately, little work has been done on the binary fraction of carbon-normal extremely metal-poor stars, and more observations are needed to study whether there is a difference in binary fraction between carbon-rich and carbonnormal extremely metal-poor environments.

\subsection{HE 0107-5240}

HE 0107-5240 is the most iron-deficient binary in our sample, and at the time of its discovery, it was the most iron-deficient star known (Christlieb et al. 2002). Since then, many different scenarios have been proposed to explain its chemical properties, including its high carbon abundance. There are two main scenarios: 1) the abundance pattern of the star reflects the interstellar medium from which it was born, which has been polluted by one or more primordial core-collapse supernovae; alternatively, 2) the surface of the star has been polluted by material from a binary companion. So far, none of the scenarios can completely explain the abundance pattern of HE 0107-5240. It might also be a combination of the two.

In the first scenario, different possible sources produce the necessary amount of carbon and the peculiar abundance pattern of HE0107-5240, for example, spinstars (Takahashi et al. 2014), faint supernovae (Umeda \& Nomoto 2003; Iwamoto et al. 2005), or a combination of normal and faint supernovae (Limongi et al. 2003). These scenarios seem to be relatively successful, but their predictions are not entirely in agreement with the observations, especially for the oxygen abundance (Bessell et al. 2004).

The alternative scenario of a binary companion transfer has been investigated by Suda et al. (2004), Lau et al. (2007) and Cruz et al. (2013). In the last work, the $s$-process abundance pattern and enhancement of carbon of HE 0107-5240 are explained by invoking mass transfer from a low-mass companion AGB star (both stars have some initial metallicity higher than zero). These models seem to fit the abundance pattern well, except for nitrogen, which is overproduced in the models. In the first two works, HE 0107-5240 starts out as a Population III (originally metalfree) star, where mass transfer from the companion star is fully responsible for the abundance pattern of HE 0107-5240. These models are also relatively successful at reproducing the chemical properties of this star. A prediction of the models is that the period of the binary is currently at least 30 years (Lau et al. 2007) and up to 150 years (Suda et al. 2004), with a maximum radial velocity variation of $6.5-7 \mathrm{~km} \mathrm{~s}^{-1}$. Their period range of 11 000-55 000 days and semi-amplitude of $\sim 3.5 \mathrm{~km} \mathrm{~s}^{-1}$ are in good agreement with the results of this work; see the first panel of Fig. 5. Additionally, Venn et al. (2014) have found a marginal detection of mid-IR excess of this star. They speculate that if this excess is real, it might be a possible indication for a debris disk formed in a binary interaction.

In summary, our observations support the binary transfer model for the origin of HE 0107-5240, where this star is potentially a true first-generation star whose pristine atmosphere has been spoiled during its lifetime. In the binary transfer scenario, it can also be a second-generation star whose surface is additionally polluted by mass-transfer from a companion, which would complicate the interpretation of its abundance pattern.

\subsection{Magnesium}

In their comparison of the two different CEMP-no sub-groups, Yoon et al. (2016) examined magnesium and found that in $\mathrm{A}(\mathrm{C})$ versus $\mathrm{A}(\mathrm{Mg})$ space, the Group II stars scale roughly with $\mathrm{A}(\mathrm{C})$, whereas the Group III stars do not and appear offset to lower $\mathrm{A}(\mathrm{Mg})$ values. Based on this behaviour (and similar behaviours for other elements), the authors suggested that the Group II and Group III CEMP-no stars could be associated with different classes of progenitors, possibly the faint mixing-and-fallback supernovae and spinstar, respectively. They reported this as a tentative conclusion, and that there may be other factors at play. Several of the CEMP-no binaries fall in the Group III subclass, suggesting that binarity may be one of those other factors.

Recently, Hartwig et al. (2018) have presented a novel diagnostic to identify second-generation stars whose birth gas cloud was enriched by only one supernova. They use the so-called divergence of the chemical displacement to identify regions in chemical space where it is likely to find this type of stars. This divergence does not depend on many assumptions in addition to the input supernovae yields for core-collapse, pair-instability, and faint supernovae. Hartwig and collaborators did not include rotating first stars or any mass-transfer scenarios as possible sources of metals in their model, but reported that they plan to add these in later work. One of the abundance spaces they identify as useful is $[\mathrm{Fe} / \mathrm{H}]$ versus $[\mathrm{Mg} / \mathrm{C}]$.

To determine what the CEMP stars look like in this space, we compiled their magnesium abundances. For the single and binary CEMP-no stars, $[\mathrm{Mg} / \mathrm{C}]$ values are listed in Table B.1. For further Group II and III stars we used the compilation of Yoon et al. (2016), which we supplemented with $[\mathrm{Mg} / \mathrm{Fe}]$ for Group I stars. For the CEMP-s stars, we compiled $[\mathrm{Mg} / \mathrm{Fe}]$ from Yong et al. (2013) and Roederer et al. (2014) to obtain a representative sample of CEMP- $s$ stars for comparison (both used the Asplund et al. 2009 solar abundances). We cross-matched these stars with Yoon et al. (2016) to derive the corrected carbon abundances for consistency. We did not include the binary information for the CEMP- $s$ stars since only a few stars with binarity information are included in the combined Yong and Roederer sample, and it can be assumed that almost all of them are in fact in binary systems.

The result is shown in Fig. 10. The CEMP-s stars all lie outside the range where Hartwig et al. (2018) claimed that mono-enrichment is likely. This is not surprising since they are expected to have received mass-transfer from a binary companion, and the models do not include this. The CEMP- $s$ stars are offset towards lower $[\mathrm{Mg} / \mathrm{C}]$ than most of the CEMP-no stars at similar $[\mathrm{Fe} / \mathrm{H}]$, which is consistent with their formation scenario of binary transfer from a former AGB companion. This transfer mainly enhances $\mathrm{C}$, not $\mathrm{Mg}$, in the companion star. This diagram may be a useful tool for uncovering stars that have undergone mass-transfer. 


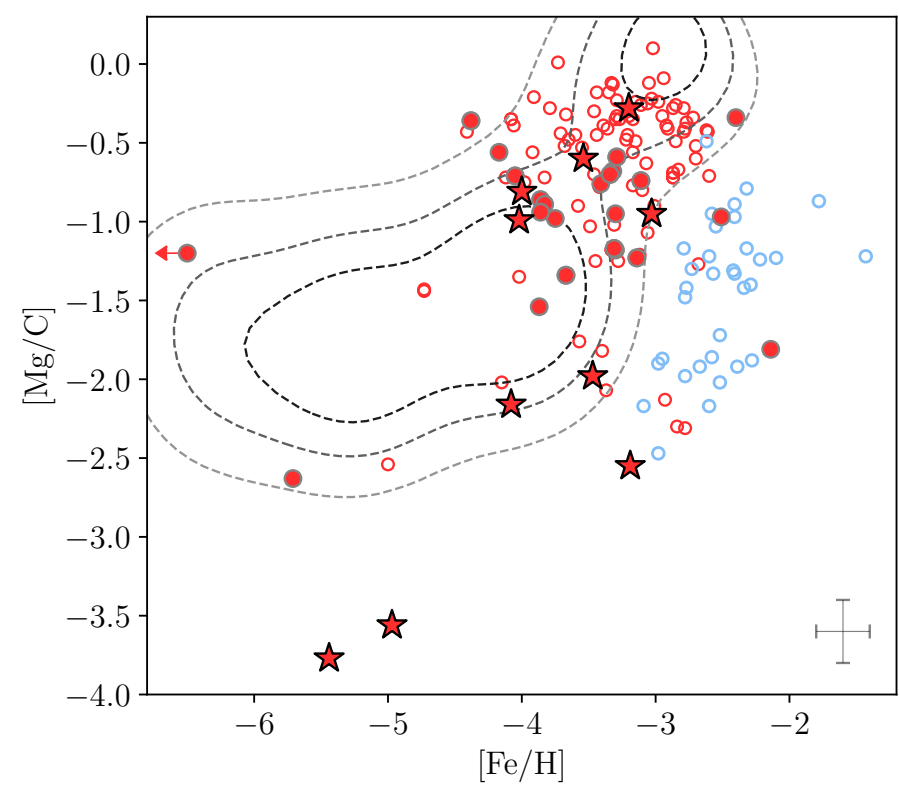

Fig. 10. Following Hartwig et al. (2018), we present $[\mathrm{Mg} / \mathrm{C}]$ values for CEMP-no and CEMP- $s$ stars against $[\mathrm{Fe} / \mathrm{H}]$. The symbols are the same as in Figs. 8 and 9, while open symbols represent stars without binarity information. Typical error bars are indicated in the lower right corner. Contours of likely mono-enrichment are over-plotted (T. Hartwig, priv. comm.).

Most of the CEMP-no stars lie in the mono-enrichment range. Five of the CEMP-no binary stars, however, have relative low values of $[\mathrm{Mg} / \mathrm{C}](\lesssim-2.0)$. Like the CEMP- $s$ stars, these stars may have experienced mass transfer from a binary companion that enhanced C, but not Mg. These five stars are HE 0107-5240, SDSS J0929+0238, G77-61, HE 1150-0428, and CS 22957-027 (in order of increasing $[\mathrm{Fe} / \mathrm{H}]$ ). For HE 0107-5240, the very low $[\mathrm{Mg} / \mathrm{C}]$ ratio was also reproduced in the mass-transfer model by Cruz et al. (2013). G77-61 most likely has a white dwarf as a companion (Dearborn et al. 1986), so that past mass transfer is also likely. For SDSS J0929+0238 we argued earlier that the masstransfer scenario is unlikely (see Sect. 5.1), but it is still possible. If it is has not experienced AGB mass transfer, some other explanation needs to be found for its low $[\mathrm{Mg} / \mathrm{C}]$.

All CEMP-no stars that overlap with the location of the CEMP- $s$ stars in this diagram are Group I stars, regardless of their binary status. CEMP-no stars in Group I have $\mathrm{A}(\mathrm{C})$ and $[\mathrm{Fe} / \mathrm{H}]$ similar to those of the CEMP- $s$ stars, but low $[\mathrm{Ba} / \mathrm{Fe}]$. The source of these high carbon abundances is unclear, especially for the single stars, but it is interesting that in this space they also share properties with CEMP- $s$ stars. However, it also shows that interpretation of this diagram is not trivial, and we should be careful to draw strong conclusions.

Finally, we emphasise that further caution should be taken when interpreting this figure, since most of the abundance measurements were not computed using non-LTE and/or 3D models, and such corrections can be important for both $\mathrm{C}$ and $\mathrm{Mg}$.

\section{Radial velocity outlook with Gaia}

More data are needed to increase the sample of CEMP-no stars with multiple radial velocity measurements and to place more stringent constraints on the orbits for several of the CEMPno binaries. This might also shed light on the evolutionary status of the companion stars and constrain mass transfer

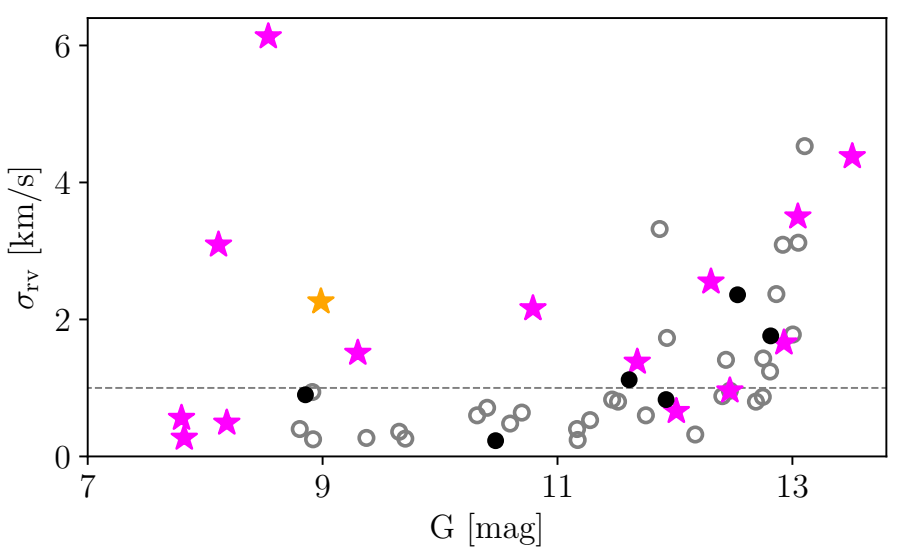

Fig. 11. Radial velocity uncertainty from Gaia vs. the Gaia G magnitude. Stars are labelled by binarity using the information from the Yoon et al. (2016) compilation, where magenta stars are binaries, black circles are non-binaries, and open grey circles represent stars of unknown binarity. The orange star is HD 135148. At fainter magnitudes, the radial velocity uncertainties in Gaia increase. The $1 \mathrm{~km} \mathrm{~s}^{-1}$ limit is marked by the grey dotted line.

models. Additionally, more long-period variable stars might be hiding in the current sample. The ESA Gaia mission will have several epochs of radial velocity data for all the brightest stars in the Galaxy down to $V \approx 16.2$ (Gaia Collaboration 2016). For the faintest metal-poor $([\mathrm{Fe} / \mathrm{H}]=-1.5)$ stars, Gaia will have endof-mission radial velocity uncertainties larger than $15 \mathrm{~km} \mathrm{~s}^{-1}$, whereas for stars brighter than $V=14$, the expected uncertainty is between $0.5-2 \mathrm{~km} \mathrm{~s}^{-15}$. This is unfortunately not the uncertainty on the individual radial velocity measurements, but that of the combined end-of-mission radial velocity. Moreover, only for the brightest stars will the single epoch radial velocities be probably released in a future Gaia data release. It is unclear how much Gaia will contribute to providing multiple good radial velocity measurements that can constrain orbits of CEMP stars. It is therefore still important to continue the radial velocity monitoring effort with high-resolution spectrographs here on Earth.

\subsection{Method}

Gaia data can, however, be used to find new binary systems, even in Gaia DR2 (Gaia Collaboration 2018). The radial velocity uncertainties provided in DR2 are the result of the combination of multiple radial velocity measurements, and if stars vary in radial velocity over the course of the Gaia observations, they will have higher radial velocity uncertainties than expected from the precision for stars of their magnitude and effective temperature. This approach is similar to what was done to investigate binarity in CEMP stars discovered in the APOGEE survey (Kielty et al. 2017).

An example of this type of analysis is shown in Fig. 11, where we cross-matched the Yoon et al. (2016) CEMP star sample with Gaia DR2 and present the uncertainty in radial velocity versus the $G$ magnitude for the stars that are sufficiently bright to be included in the current data release. At the faintest end, the precision is expected to be a maximum of $\sim 2 \mathrm{~km} \mathrm{~s}^{-1}$ for the hottest stars in our sample, and at the bright end, the precision should be less than $1 \mathrm{~km} \mathrm{~s}^{-1}$ (Katz et al. 2018). In the figure we indicate binarity information from the compilation, where the magenta stars are known

5 https://www.cosmos.esa.int/web/gaia/rvsperformance 
A. Arentsen et al.: Binarity among CEMP-no stars: an indication of multiple formation pathways?

Table 2. CEMP stars that have Gaia radial velocity uncertainties $>1 \mathrm{~km} \mathrm{~s}^{-1}$, with new binary candidates in bold-face.

\begin{tabular}{|c|c|c|c|c|c|c|c|c|c|c|c|c|}
\hline Name & $\begin{array}{l}\text { CEMP } \\
\text { class }\end{array}$ & $\begin{array}{r}\mathrm{G} \\
(\mathrm{mag})\end{array}$ & $\begin{array}{r}r v_{\text {Gaia }} \\
\left(\mathrm{km} \mathrm{s}^{-1}\right)\end{array}$ & $\begin{array}{r}\sigma_{\text {Gaia }} \\
\left(\mathrm{km} \mathrm{s}^{-1}\right) \\
\end{array}$ & $\begin{array}{r}r v_{\text {lit }}{ }^{a} \\
\left(\mathrm{~km} \mathrm{~s}^{-1}\right)\end{array}$ & $\begin{array}{r}\sigma_{\text {lit }} \\
\left(\mathrm{km} \mathrm{s}^{-1}\right)\end{array}$ & $n_{\text {lit }}$ & Ref. & $\begin{array}{r}T_{\text {eff }}^{b} \\
(\mathrm{~K})\end{array}$ & {$[\mathrm{Fe} / \mathrm{H}]^{c}$} & $\begin{array}{r}T_{\text {eff, }} \\
(\mathrm{K})\end{array}$ & {$[\mathrm{Fe} / \mathrm{H}]_{\mathrm{T}}$} \\
\hline HE 2155-3750 & $s$ & 13.1 & 77.5 & 4.5 & 27.7 & $10.0^{b}$ & 1 & 1 & 5000 & -2.64 & 5500 & 0.0 \\
\hline HE 1305+0007 & $s$ & 11.9 & 228.5 & 3.3 & 217.8 & 1.5 & 1 & 2 & 4750 & -2.08 & 4750 & -2.0 \\
\hline HE 2123-0329 & no & 13.1 & -217.7 & 3.1 & -219.1 & 0.4 & 2 & 3 & 4725 & -3.22 & 6500 & -1.5 \\
\hline SMSS J1738-1457 & no & 12.9 & 43.7 & 3.1 & -26.9 & 2.0 & 1 & 4 & 4600 & -3.58 & 5700 & 0.0 \\
\hline HKII 17435-00532 & $r / s$ & 12.8 & 39.3 & 2.4 & 38.9 & 0.3 & 4 & 5 & 5200 & -2.23 & 6000 & -1.5 \\
\hline CS 30301-015 & $s$ & 12.8 & 87.0 & 1.8 & 86.6 & 0.1 & 18 & 6 & 4900 & -2.73 & 6000 & -1.5 \\
\hline HE 2319-5228 & no & 13.0 & 286.2 & 1.8 & 294.3 & 4.0 & 2 & 7 & 4900 & -2.60 & 6000 & -1.5 \\
\hline BS 16077-077 & $s$ & 11.9 & 68.3 & 1.7 & $\cdots$ & $\ldots$ & .. & $\cdots$ & 5900 & -2.05 & 6000 & -1.5 \\
\hline CS 22947-187 & $s$ & 12.8 & -252.3 & 1.4 & -251.9 & 0.9 & 2 & 8,9 & 5300 & -2.58 & 6000 & -1.5 \\
\hline HE $1305+0132$ & $s$ & 12.4 & 157.0 & 1.4 & $\ldots$ & $\ldots$ & .. & $\ldots$ & 4462 & -2.45 & 4500 & 0.0 \\
\hline CS 22873-128 & no & 12.8 & 207.15 & 1.2 & 205.5 & 0.3 & 2 & 8,9 & 4710 & -3.32 & 6000 & -1.5 \\
\hline
\end{tabular}

Notes. ${ }^{(a)}$ For stars with only one measurement, this is the radial velocity as presented in the literature. For stars with multiple measurements (see column $n_{\text {lit }}$ ), it is the (weighted) average of the different measurements. Similarly, for the uncertainty, it is either the uncertainty reported in the literature or the (weighted) standard deviation between different literature values. ${ }^{(b)}$ As reported in the Yoon et al. (2016) compilation. ${ }^{(c)}$ No uncertainty is given in the reference, but since it is a measurement from an intermediate-resolution spectrum, we assume $\sigma=10 \mathrm{~km} \mathrm{~s}^{-1}$.

References. (radial velocities) (1) Placco et al. (2011), (2) Goswami et al. (2006), (3) Hollek et al. (2011), (4) Jacobson et al. (2015), (5) Roederer et al. (2008), (6) Hansen et al. (2016a), (7) Beers et al. (2017), (8) Roederer et al. (2014), and (9) McWilliam et al. (1995).

binaries, the black circles are known non-binaries, and open grey symbols represent stars of unknown binarity. The orange star in the Figure is HD 135148, for which the binary information was not provided in the Yoon et al. (2016) compilation. We "rediscovered" this binary using the Gaia information and found that it was already known from Carney et al. (2003). It is clear that several of the binaries indeed have larger radial velocity uncertainties than expected from the precision alone.

\subsection{New CEMP binary candidates}

We investigated stars of unknown binarity with high $\sigma_{\text {Gaia }}$ values $\left(>1 \mathrm{~km} \mathrm{~s}^{-1}\right)$, see a summary in Table 2 . We included the $T_{\text {eff,T }}$ and $[\mathrm{Fe} / \mathrm{H}]_{\mathrm{T}}$ of the template that was used in the Gaia radial velocity determination. A poor template with an incorrect shape of the spectral lines might not necessarily result in a poor radial velocity, but will most probably influence the radial velocity uncertainty. The standard Gaia radial velocity templates have $[\mathrm{Fe} / \mathrm{H}]$ of either 0.0 or -1.5 , except when the metallicity of the star is known (Katz et al. 2018). Even though most of our stars are more metal-poor than $[\mathrm{Fe} / \mathrm{H}]=-1.5$, the radial velocity precision seems to be good with the $[\mathrm{Fe} / \mathrm{H}]=-1.5$ templates, since for several stars in Table 2 the literature and Gaia radial velocities agree to within $1 \mathrm{~km} \mathrm{~s}^{-1}$. This is even the case for stars where $T_{\text {eff,T }}$ differs by more than $1000 \mathrm{~K}$ from the literature (see CS 30301-015 and CS 22873-128). It is unclear how good the velocities are with the $[\mathrm{Fe} / \mathrm{H}]=0.0$ templates, since for two stars with such a template the literature radial velocities are highly discrepant with those from Gaia. In future Gaia data releases the stellar parameters from the Gaia spectra (Bp, Rp, and RVS) will be used to select better radial velocity templates, which should reduce the mismatch between observations and templates and improve the radial velocity quality (Katz et al. 2018).

In the cross-match between the Yoon et al. (2016) CEMP sample and the Gaia data are two CEMP-s stars that are good new binary candidates: HE 2155-3750 and HE 1305+0007. They have large $\sigma_{\text {Gaia }}$ and large discrepancies $\left(>10 \mathrm{~km} \mathrm{~s}^{-1}\right)$ between the Gaia radial velocity and the literature. The $[\mathrm{Fe} / \mathrm{H}]_{\mathrm{T}}$ of HE 2155-3750 is a poor match, which may cause part of the discrepancy, but probably not the full $\sim 50 \mathrm{~km} \mathrm{~s}^{-1}$. These two stars seem good binary candidates.

Additionally, and of more interest for this work, two CEMPno stars seem to vary in radial velocity: SMSS J1738-1457 and HE 2319-5228. The applied radial velocity template for SMSS J1738-1457 is a poor match in both $[\mathrm{Fe} / \mathrm{H}]$ and $T_{\text {eff. }}$ It is unlikely, however, that this causes a difference of $\sim 60 \mathrm{~km} \mathrm{~s}^{-1}$. This star is therefore still a good binary candidate. With its $\mathrm{A}(\mathrm{C})=6.18$ and $[\mathrm{Fe} / \mathrm{H}]=-3.58$ (Jacobson et al. 2015), it lies on the lower carbon band. Then HE 2319-5228 shows a modest radial velocity variation of $\sim 8 \mathrm{~km} \mathrm{~s}^{-1}$. We showed that a mismatch between $T_{\text {eff }}$ and $T_{\text {eff, } T}$ is not likely to cause such large differences (e.g. compare to CS 30301-015), therefore this star remains a good binary candidate. It has $\mathrm{A}(\mathrm{C})=6.51$ and $[\mathrm{Fe} / \mathrm{H}]=-3.4$ (Beers et al. 2017), therefore it also lies on the lower carbon band.

Another way to find binary stars using Gaia is through the astrometric excess noise $(D)$ and the goodness of fit of the Gaia astrometry. This selects stars with poor astrometric solutions that are caused by a companion star (as demonstrated e.g. in Evans 2018). This method works best for nearby stars, and unfortunately, most of the extremely metal-poor CEMP-no stars are too far away. However, two CEMP-no stars in the Yoon et al. (2016) sample, G77-61 and CS 22958-042, have significant astrometric excess noise $(D>3)$. G77-61 is a nearby, high proper motion, known binary star with a poor goodness of fit and $D=207.9$. CS 22958-042 has $D=9.7$ and also a poor goodness of fit, therefore this star might be in a binary system as well.

The analysis in this section shows that Gaia can be used to find new candidate binary CEMP systems. Follow-up spectroscopy is still needed, however, to verify the results and characterise the orbits of the new binary systems.

\section{Conclusions}

We have discussed the results of extending a radial velocity monitoring program for CEMP-no stars that started with Starkenburg et al. (2014). We identified four new CEMP-no stars in binary systems based on their radial velocity variations. 
Together with stars from the literature, we now have a sample of 11 CEMP-no binaries and 23 likely single CEMPno stars, resulting in a binary fraction of $32_{-9}^{+10} \%$. This only marginally agrees with the previous estimate for CEMP-no stars by Hansen et al. (2016b), which was similar to the binary fraction of metal-poor carbon-normal giants $\left(16_{-4}^{+5} \%\right.$, Carney et al. 2003).

The periods of the CEMP-no binaries are similar to the typical periods of CEMP- $s$ stars, ranging from several 100 to several 1000 days for most of the stars. It applies to all the binaries in our sample that if the companion of the main star is currently a white dwarf, it is probable that the current CEMP-no star has been polluted during the AGB phase of the companion. This enhances the amount of carbon in the star and changes its abundance pattern. None of the CEMP-no binary stars show a clear enhancement in $[\mathrm{Ba} / \mathrm{Fe}]$ that would be indicative of $s$-process element transfer. Therefore, if binary transfer from an AGB companion has occurred, it must have been an extremely metal-poor AGB star that has not produced a significant amount of $s$-process elements.

Our small CEMP-no radial velocity sample shows an apparent difference in binary fraction between the CEMP-no stars with lower $\mathrm{A}(\mathrm{C})\left(18_{-9}^{+14} \%\right)$ and those with higher $\mathrm{A}(\mathrm{C})\left(47_{-14}^{+15} \%\right)$. This higher binary fraction of stars with high absolute carbon abundances can have interesting implications for the origins of CEMP-no stars. We propose that some of the high $\mathrm{A}(\mathrm{C})$ binaries started out as carbon-normal stars or CEMP-no stars on the lowcarbon band and received extra carbon from a companion, which moved them to the intermediate- to high-carbon band. Alternatively, star formation might have a tendency to form more binaries at high $\mathrm{A}(\mathrm{C})$ or extremely low $[\mathrm{Fe} / \mathrm{H}]$.

The detection of radial velocity variations in the hypermetal-poor star HE 0107-5240 is very interesting. Some models have tried to explain its abundance pattern based on the assumption that its completely pristine surface has been polluted by a former AGB companion, which currently is a white dwarf. Previous to this current detection, there was no clear evidence for its variability in radial velocity. This makes the binary formation scenario as probable as or even more probable than scenarios invoking faint supernovae or spinstars.

Further monitoring of our new CEMP-no binary stars would allow deriving better orbit parameters, which is necessary to constrain possible mass transfer models. Monitoring of additional stars is needed to investigate wether there truly is a larger fraction of CEMP-no binary stars among stars with high absolute carbon abundance and/or extremely low metallicity. Although Gaia may not (yet) be instrumental in studying specific binary systems in detail or in constraining the binary fraction, it shows promise in discovering new binary systems. We have highlighted some new binary candidates.

Some (although not necessarily all) CEMP-no binary stars might have been polluted by a companion star, which affects and complicates the interpretation of their abundance patterns. Their abundances may not only be probes of faint supernovae and/or spinstars, but also of extremely metal-poor AGB stars.

Acknowledgements. AA and ES gratefully acknowledge funding by the Emmy Noether program from the Deutsche Forschungsgemeinschaft (DFG). KAV thanks the National Science and Engineering Research Council for partial funding through their Discovery Grants program. We thank Lison Malo for her help with the CFHT ESPaDOnS spectra, Norbert Christlieb for sharing his radial velocity data for HE 0557-4840 and HE 0107-5240 with us, Terese Hansen for her re-determination of the stellar parameters for HE 0219-1739 and Tilman Hartwig for sharing his mono-enrichment contours for Fig. 10. We thank John Norris, Jay Farihi and Terese Hansen for their useful comments on a draft of this work. The observations reported in this paper were obtained with the
Southern African Large Telescope (SALT) and the Canada France Hawaii Telescope (CFHT). It also made use of data obtained from the ESO Science Archive Facility under request numbers aarentsen 312315 and 358299. This work has made use of data from the European Space Agency (ESA) mission Gaia (https://www.cosmos.esa.int/gaia), processed by the Gaia Data Processing and Analysis Consortium (DPAC, https://www.cosmos.esa.int/ web/gaia/dpac/consortium). Funding for the DPAC has been provided by national institutions, in particular the institutions participating in the Gaia Multilateral Agreement. This research made extensive use of the MATPLOTLIB (Hunter 2007) and PANDAs (McKinney 2010) Python packages.

\section{References}

Abate, C., Pols, O. R., Karakas, A. I., \& Izzard, R. G. 2015, A\&A, 576, A118 Aguado, D. S., González Hernández, J. I., Allende Prieto, C., \& Rebolo, R. 2018 ApJ, 852, L20

Allende Prieto, C., Fernández-Alvar, E., Aguado, D. S., et al. 2015, A\&A, 579, A98

Alvarez, R., \& Plez, B. 1998, A\&A, 330, 1109

Aoki, W., Beers, T. C., Lee, Y. S., et al. 2013, AJ, 145, 13

Asplund, M., Grevesse, N., Sauval, A. J., \& Scott, P. 2009, ARA\&A, 47, 48

Bandyopadhyay, A., Sivarani, T., Susmitha, A., et al. 2018, ApJ, 859, 114

Barklem, P. S., Christlieb, N., Beers, T. C., et al. 2005, A\&A, 439, 129

Beers, T. C., \& Christlieb, N. 2005, ARA\&A, 43, 531

Beers, T. C., Preston, G. W., \& Shectman, S. A. 1992, AJ, 103, 1987

Beers, T. C., Placco, V. M., Carollo, D., et al. 2017, ApJ, 835, 81

Bessell, M. S., Christlieb, N., \& Gustafsson, B. 2004, ApJ, 612, L61

Bessell, M. S., Collet, R., Keller, S. C., et al. 2015, ApJ, 806, L16

Bonifacio, P., Caffau, E., Spite, M., et al. 2015, A\&A, 579, A28

Bonifacio, P., Caffau, E., Spite, M., et al. 2018, A\&A, 612, A65

Bramall, D. G., Sharples, R., Tyas, L., et al. 2010, in Ground-based and Airborne Instrumentation for Astronomy III, Proc. SPIE, 7735, 77354F

Bramall, D. G., Schmoll, J., Tyas, L. M. G., et al. 2012, in Ground-based and Airborne Instrumentation for Astronomy IV, Proc. SPIE, 8446, 84460A

Buckley, D. A. H., Swart, G. P., \& Meiring, J. G. 2006, in Society of PhotoOptical Instrumentation Engineers (SPIE) Conference Series, Proc. SPIE, $6267,62670 Z$

Busso, M., Gallino, R., \& Wasserburg, G. J. 1999, ARA\&A, 37, 239

Caffau, E., Bonifacio, P., François, P., et al. 2011, Nature, 477, 67

Caffau, E., Bonifacio, P., Spite, M., et al. 2016, A\&A, 595, L6

Caffau, E., Bonifacio, P., Starkenburg, E., et al. 2017, Astron. Nachr., 338, 686

Carney, B. W., Latham, D. W., Stefanik, R. P., Laird, J. B., \& Morse, J. A. 2003, AJ, 125, 293

Carney, B. W., Latham, D. W., Stefanik, R. P., \& Laird, J. B. 2008, AJ, 135, 196

Chiaki, G., Tominaga, N., \& Nozawa, T. 2017, MNRAS, 472, L115

Chiappini, C. 2013, Astron. Nachr., 334, 595

Choi, J., Dotter, A., Conroy, C., et al. 2016, ApJ, 823, 102

Choplin, A., Hirschi, R., Meynet, G., \& Ekström, S. 2017, A\&A, 607, L3

Christlieb, N., Bessell, M. S., Beers, T. C., et al. 2002, Nature, 419, 904

Christlieb, N., Beers, T. C., Barklem, P. S., et al. 2004, A\&A, 428, 1027

Cohen, J. G., Christlieb, N., Thompson, I., et al. 2013, ApJ, 778, 56

Crause, L. A., Sharples, R. M., Bramall, D. G., et al. 2014, in Ground-based and Airborne Instrumentation for Astronomy V, Proc. SPIE, 9147, 91476T

Cruz, M. A., Serenelli, A., \& Weiss, A. 2013, A\&A, 559, A4

Dearborn, D. S. P., Liebert, J., Aaronson, M., et al. 1986, ApJ, 300, 314

Donati, J. F. 2003, in Solar Polarization, eds. J. Trujillo-Bueno, \& J. Sanchez Almeida, ASP Conf. Ser., 307, 41

Donati, J. F., Semel, M., Carter, B. D., Rees, D. E., \& Collier Cameron, A. 1997, MNRAS, 291, 658

Dotter, A. 2016, ApJS, 222, 8

Evans, D. F. 2018, Res. Notes Am. Astron. Soc., 2, 20

Foreman-Mackey, D. 2016, J. Open Sou. Softw., 1

Frebel, A., Collet, R., Eriksson, K., Christlieb, N., \& Aoki, W. 2008, ApJ, 684, 588

Frebel, A., Chiti, A., Ji, A. P., Jacobson, H. R., \& Placco, V. M. 2015, ApJ, 810, L27

Gaia Collaboration (Prusti, T., et al.) 2016, A\&A, 595, A1

Gaia Collaboration (Brown, A. G. A., et al.) 2018, A\&A, 616, A1

Gil-Pons, P., Doherty, C. L., Gutiérrez, J. L., et al. 2018, PASA, accepted [arXiv:1810.00982]

Giridhar, S., Lambert, D. L., Gonzalez, G., \& Pandey, G. 2001, PASP, 113, 519

Goswami, A., Aoki, W., Beers, T. C., et al. 2006, MNRAS, 372, 343

Gustafsson, B., Edvardsson, B., Eriksson, K., et al. 2008, A\&A, 486, 951

Hansen, T., Hansen, C. J., Christlieb, N., et al. 2015, ApJ, 807, 173

Hansen, T. T., Andersen, J., Nordström, B., et al. 2016a, A\&A, 588, A3

Hansen, T. T., Andersen, J., Nordström, B., et al. 2016b, A\&A, 586, A160 
A. Arentsen et al.: Binarity among CEMP-no stars: an indication of multiple formation pathways?

Hartwig, T., Yoshida, N., Magg, M., et al. 2018, MNRAS, 478, 1795

Hernitschek, N., Schlafly, E. F., Sesar, B., et al. 2016, ApJ, 817, 73

Hollek, J. K., Frebel, A., Roederer, I. U., et al. 2011, ApJ, 742, 54

Hunter, J. D. 2007, Comput. Sci. Eng., 9, 90

Ito, H., Aoki, W., Beers, T. C., et al. 2013, ApJ, 773, 33

Iwamoto, N., Umeda, H., Tominaga, N., Nomoto, K., \& Maeda, K. 2005, Science, 309, 451

Jacobson, H. R., Keller, S., Frebel, A., et al. 2015, ApJ, 807, 171

Karakas, A. I., \& Lattanzio, J. C. 2014, PASA, 31, e030

Katz, D., Sartoretti, P., Cropper, M., et al. 2018, A\&A, submitted [arXiv:1804.09372]

Keller, S. C., Bessell, M. S., Frebel, A., et al. 2014, Nature, 506, 463

Kielty, C. L., Venn, K. A., Loewen, N. B., et al. 2017, MNRAS, 471, 404

Latham, D. W., Stefanik, R. P., Torres, G., et al. 2002, AJ, 124, 1144

Lau, H. H. B., Stancliffe, R. J., \& Tout, C. A. 2007, MNRAS, 378, 563

Lee, Y. S., Beers, T. C., Masseron, T., et al. 2013, AJ, 146, 132

Limongi, M., Chieffi, A., \& Bonifacio, P. 2003, ApJ, 594, L123

Lucatello, S., Tsangarides, S., Beers, T. C., et al. 2005, ApJ, 625, 825

Lugaro, M., Karakas, A. I., Stancliffe, R. J., \& Rijs, C. 2012, ApJ, 747, 2

Manset, N., \& Donati, J. F. 2003, in Polarimetry in Astronomy, ed. S. Fineschi, Proc. SPIE, 4843, 425

Matsuno, T., Aoki, W., Suda, T., \& Li, H. 2017, PASJ, 69, 24

McClure, R. D., \& Woodsworth, A. W. 1990, ApJ, 352, 709

McKinney, W. 2010, in Proceedings of the 9th Python in Science Conference, eds. S. van der Walt, \& J. Millman, 51

McWilliam, A., Preston, G. W., Sneden, C., \& Shectman, S. 1995, AJ, 109, 2736

Meynet, G., Ekström, S., \& Maeder, A. 2006, A\&A, 447, 623

Meynet, G., Hirschi, R., Ekstrom, S., et al. 2010, A\&A, 521, A30

Moe, M., Kratter, K. M., \& Badenes, C. 2018, ApJ, submitted [arXiv:1808.02116]

Nomoto, K., Kobayashi, C., \& Tominaga, N. 2013, ARA\&A, 51, 457

Nordlander, T., Amarsi, A. M., Lind, K., et al. 2017, A\&A, 597, A6

Norris, J. E., Ryan, S. G., \& Beers, T. C. 1997, ApJ, 488, 350

Norris, J. E., Christlieb, N., Korn, A. J., et al. 2007, ApJ, 670, 774
Norris, J. E., Wyse, R. F. G., Gilmore, G., et al. 2010, ApJ, 723, 1632

Norris, J. E., Yong, D., Bessell, M. S., et al. 2013a, ApJ, 762, 28

Norris, J. E., Bessell, M. S., Yong, D., et al. 2013b, ApJ, 762, 25

Placco, V. M., Kennedy, C. R., Beers, T. C., et al. 2011, AJ, 142, 188

Placco, V. M., Frebel, A., Beers, T. C., \& Stancliffe, R. J. 2014, ApJ, 797, 21

Placco, V. M., Beers, T. C., Reggiani, H., \& Meléndez, J. 2016a, ApJ, 829, L24

Placco, V. M., Frebel, A., Beers, T. C., et al. 2016b, ApJ, 833, 21

Plez, B. 2008, Phys. Scr. Vol. T, 133, 014003

Plez, B., \& Cohen, J. G. 2005, A\&A, 434, 1117

Preston, G. W., \& Sneden, C. 2001, AJ, 122, 1545

Price-Whelan, A., \& Hogg, D. W. 2017, adrn/thejoker: Release v0.1, https://zenodo.org/record/264481

Price-Whelan, A. M., Hogg, D. W., Foreman-Mackey, D., \& Rix, H.-W. 2017, ApJ, 837, 20

Roederer, I. U., Frebel, A., Shetrone, M. D., et al. 2008, ApJ, 679, 1549

Roederer, I. U., Preston, G. W., Thompson, I. B., et al. 2014, AJ, 147, 136

Schlaufman, K. C., Thompson, I. B., \& Casey, A. R. 2018, ApJ, 867, 98

Simmerer, J., Sneden, C., Cowan, J. J., et al. 2004, ApJ, 617, 1091

Spite, M., Caffau, E., Bonifacio, P., et al. 2013, A\&A, 552, A107

Starkenburg, E., Shetrone, M. D., McConnachie, A. W., \& Venn, K. A. 2014, MNRAS, 441, 1217

Starkenburg, E., Martin, N., Youakim, K., et al. 2017, MNRAS, 471, 2587

Starkenburg, E., Aguado, D. S., Bonifacio, P., et al. 2018, MNRAS, 481, 3838

Suda, T., Aikawa, M., Machida, M. N., Fujimoto, M. Y., \& Iben, Jr., I. 2004, ApJ, 611, 476

Takahashi, K., Umeda, H., \& Yoshida, T. 2014, ApJ, 794, 40

Tominaga, N., Iwamoto, N., \& Nomoto, K. 2014, ApJ, 785, 98

Tonry, J., \& Davis, M. 1979, AJ, 84, 1511

Umeda, H., \& Nomoto, K. 2003, Nature, 422, 871

Umeda, H., \& Nomoto, K. 2005, ApJ, 619, 427

Venn, K. A., Puzia, T. H., Divell, M., et al. 2014, ApJ, 791, 98

Yong, D., Norris, J. E., Bessell, M. S., et al. 2013, ApJ, 762, 26

Yoon, J., Beers, T. C., Placco, V. M., et al. 2016, ApJ, 833, 20 


\section{Appendix A: Measured radial velocities}

Table A.1. Radial velocities determined from the CFHT and SALT spectra in this work.

\begin{tabular}{|c|c|c|c|c|}
\hline Name & $\begin{array}{r}r v \\
\left(\mathrm{~km} \mathrm{~s}^{-1}\right)\end{array}$ & $\begin{array}{r}\text { Err } \\
\left(\mathrm{km} \mathrm{s}^{-1}\right)\end{array}$ & $\begin{array}{r}\text { HJD } \\
-2450000\end{array}$ & Flag $^{a}$ \\
\hline $\mathrm{BD}+44^{\circ} 493$ & -149.77 & 0.55 & 6525.58 & $\mathrm{C}$ \\
\hline $\mathrm{BD}+44^{\circ} 493$ & -150.24 & 0.51 & 6549.55 & $\mathrm{C}$ \\
\hline $\mathrm{BD}+44^{\circ} 493$ & -149.95 & 0.35 & 6697.25 & $\mathrm{C}$ \\
\hline $\mathrm{BD}+44^{\circ} 493$ & -149.32 & 1.32 & 6709.21 & $\mathrm{C}$ \\
\hline $\mathrm{BD}+44^{\circ} 493$ & -149.62 & 1.13 & 6709.21 & $\mathrm{C}$ \\
\hline BS 16929-005 & -50.84 & 0.54 & 6523.22 & $\mathrm{C}$ \\
\hline BS 16929-005 & -50.56 & 0.76 & 6675.68 & $\mathrm{C}$ \\
\hline BS 16929-005 & -51.27 & 0.90 & 6701.62 & $\mathrm{C}$ \\
\hline BS 16929-005 & -49.70 & 0.60 & 6701.63 & $\mathrm{C}$ \\
\hline CS 22878-027 & -91.77 & 0.35 & 6520.32 & $\mathrm{C}$ \\
\hline CS 22878-027 & -91.45 & 0.34 & 6530.23 & $\mathrm{C}$ \\
\hline CS 22878-027 & -91.56 & 0.51 & 6700.64 & $\mathrm{C}$ \\
\hline CS 22878-027 & -91.62 & 0.34 & 6812.32 & $\mathrm{C}$ \\
\hline CS 22949-037 & -126.10 & 0.44 & 6521.53 & $\mathrm{C}$ \\
\hline CS 22949-037 & -126.12 & 0.41 & 6532.54 & $\mathrm{C}$ \\
\hline CS 22949-037 & -126.05 & 0.56 & 6549.50 & $\mathrm{C}$ \\
\hline CS 22949-037 & -125.67 & 0.32 & 6816.59 & $\mathrm{C}$ \\
\hline CS 22949-037 & -126.10 & 0.36 & 6824.54 & $\mathrm{C}$ \\
\hline CS 22957-027 & -61.43 & 0.94 & 6519.53 & $\mathrm{C}$ \\
\hline CS 22957-027 & -62.34 & 0.89 & 6532.55 & $\mathrm{C}$ \\
\hline CS 22957-027 & -61.46 & 0.92 & 6549.52 & $\mathrm{C}$ \\
\hline CS 22957-027 & -71.68 & 1.05 & 6816.61 & $\mathrm{C}$ \\
\hline CS 22957-027 & -72.09 & 0.98 & 6824.56 & $\mathrm{C}$ \\
\hline CS 29498-043 & -30.04 & 3.88 & 6609.25 & $\mathrm{~S}$ \\
\hline CS 29498-043 & -31.78 & 1.32 & 6876.25 & $\mathrm{~S}$ \\
\hline CS 29498-043 & -31.61 & 2.70 & 6968.25 & $\mathrm{~S}$ \\
\hline CS 29498-043 & -32.24 & 0.36 & 7121.64 & $\mathrm{~S}$ \\
\hline CS 29502-092 & -67.33 & 0.35 & 6519.53 & $\mathrm{C}$ \\
\hline CS 29502-092 & -66.91 & 0.38 & 6530.43 & $\mathrm{C}$ \\
\hline CS 29502-092 & -67.55 & 0.33 & 6548.33 & $\mathrm{C}$ \\
\hline CS 29502-092 & -66.93 & 0.31 & 6770.64 & $\mathrm{C}$ \\
\hline CS 29502-092 & -67.54 & 0.35 & 6770.64 & $\mathrm{C}$ \\
\hline CS 29502-092 & -66.54 & 0.26 & 6816.58 & $\mathrm{C}$ \\
\hline HE 0057-5959 & 377.90 & 3.98 & 6611.50 & $\mathrm{~S}$ \\
\hline HE $0057-5959$ & 378.23 & 1.47 & 6886.50 & $\mathrm{~S}$ \\
\hline HE $0107-5240$ & 48.15 & 0.49 & 6618.50 & $\mathrm{~S}$ \\
\hline HE $0107-5240$ & 46.60 & 4.75 & 6858.50 & $\mathrm{~S}$ \\
\hline HE $0107-5240$ & 47.19 & 2.39 & 6957.25 & $\mathrm{~S}$ \\
\hline HE 0107-5240 & 48.29 & 0.94 & 6990.50 & $\mathrm{~S}$ \\
\hline HE $0557-4840$ & 209.11 & 2.00 & 6644.25 & $\mathrm{~S}$ \\
\hline HE $0557-4840$ & 212.33 & 0.58 & 6923.50 & $\mathrm{~S}$ \\
\hline HE $1012-1540$ & 226.17 & 0.64 & 6669.61 & $\mathrm{C}$ \\
\hline HE $1012-1540$ & 225.33 & 0.59 & 6669.62 & $\mathrm{C}$ \\
\hline HE $1012-1540$ & 225.27 & 0.34 & 6675.60 & $\mathrm{C}$ \\
\hline HE $1012-1540$ & 225.35 & 0.33 & 6697.51 & $\mathrm{C}$ \\
\hline HE $1012-1540$ & 225.41 & 0.26 & 6758.22 & $\mathrm{C}$ \\
\hline HE $1012-1540$ & 225.43 & 0.49 & 6998.50 & $\mathrm{~S}$ \\
\hline
\end{tabular}

Notes. ${ }^{(a)} \mathrm{C}$ is observed with CFHT, S is observed with SALT.
Table A.1. continued.

\begin{tabular}{|c|c|c|c|c|}
\hline Name & $\begin{array}{r}r v \\
\left(\mathrm{~km} \mathrm{~s}^{-1}\right)\end{array}$ & $\begin{array}{r}\text { Err } \\
\left(\mathrm{km} \mathrm{s}^{-1}\right)\end{array}$ & $\begin{array}{r}\text { HJD } \\
-2450000\end{array}$ & Flag $^{a}$ \\
\hline HE $1150-0428$ & 38.17 & 1.70 & 6669.64 & $\mathrm{C}$ \\
\hline HE $1150-0428$ & 36.31 & 1.38 & 6675.62 & $\mathrm{C}$ \\
\hline HE $1150-0428$ & 35.90 & 1.59 & 6697.53 & $\mathrm{C}$ \\
\hline HE $1150-0428$ & 46.23 & 1.51 & 6770.44 & $\mathrm{C}$ \\
\hline HE $1150-0428$ & 54.06 & 1.55 & 6812.25 & $\mathrm{C}$ \\
\hline HE $1150-0428$ & 54.49 & 1.62 & 6817.23 & $\mathrm{C}$ \\
\hline HE $1150-0428$ & 55.91 & 1.65 & 6823.24 & $\mathrm{C}$ \\
\hline HE $1201-1512$ & 239.80 & 1.32 & 6669.67 & $\mathrm{C}$ \\
\hline HE $1201-1512$ & 237.06 & 1.70 & 6669.67 & $\mathrm{C}$ \\
\hline HE $1201-1512$ & 240.11 & 0.52 & 6697.56 & $\mathrm{C}$ \\
\hline HE $1201-1512$ & 240.07 & 0.79 & 6760.26 & $\mathrm{C}$ \\
\hline HE $1201-1512$ & 235.18 & 3.34 & 7058.47 & $\mathrm{~S}$ \\
\hline HE $1201-1512$ & 239.26 & 6.04 & 7141.24 & $\mathrm{~S}$ \\
\hline HE $1300+0157$ & 75.40 & 1.06 & 6520.22 & $\mathrm{C}$ \\
\hline HE $1300+0157$ & 72.89 & 1.20 & 6675.67 & $\mathrm{C}$ \\
\hline HE $1300+0157$ & 74.60 & 0.44 & 6700.53 & $\mathrm{C}$ \\
\hline HE $1300+0157$ & 74.67 & 0.72 & 6760.28 & $\mathrm{C}$ \\
\hline HE $1327-2326$ & 65.35 & 1.60 & 6781.25 & $\mathrm{~S}$ \\
\hline HE 1327-2326 & 64.15 & 1.35 & 7060.50 & $\mathrm{~S}$ \\
\hline HE $1327-2326$ & 67.86 & 3.52 & 7140.53 & $\mathrm{~S}$ \\
\hline HE $1506-0113$ & -93.91 & 0.54 & 6520.24 & $\mathrm{C}$ \\
\hline HE $1506-0113$ & -92.80 & 0.46 & 6527.22 & $\mathrm{C}$ \\
\hline HE $1506-0113$ & -92.75 & 0.51 & 6532.22 & $\mathrm{C}$ \\
\hline HE $1506-0113$ & -87.43 & 0.46 & 6697.63 & $\mathrm{C}$ \\
\hline HE $1506-0113$ & -88.23 & 0.46 & 6701.64 & $\mathrm{C}$ \\
\hline HE 1506-0113 & -85.32 & 0.34 & 6812.29 & $\mathrm{C}$ \\
\hline HE 1506-0113 & -84.61 & 0.37 & 6823.27 & $\mathrm{C}$ \\
\hline HE 1506-0113 & -77.13 & 1.97 & 7140.54 & $\mathrm{~S}$ \\
\hline HE 2139-5432 & 98.58 & 4.00 & 6620.25 & $\mathrm{~S}$ \\
\hline HE 2139-5432 & 95.79 & 0.29 & 6900.25 & $\mathrm{~S}$ \\
\hline HE 2142-5656 & 104.24 & 0.82 & 6609.25 & $\mathrm{~S}$ \\
\hline HE 2142-5656 & 104.06 & 1.98 & 6876.25 & $\mathrm{~S}$ \\
\hline HE 2142-5656 & 105.32 & 2.25 & 6968.25 & $S$ \\
\hline HE 2202-4831 & 56.58 & 1.20 & 6609.25 & $\mathrm{~S}$ \\
\hline HE 2202-4831 & 57.50 & 2.00 & 6990.25 & $\mathrm{~S}$ \\
\hline HE 2247-7400 & 6.54 & 1.53 & 6881.50 & $\mathrm{~S}$ \\
\hline SDSS J0140+2344 & -190.32 & 1.09 & 6525.55 & $\mathrm{C}$ \\
\hline SDSS J0140+2344 & -201.03 & 1.25 & 6549.52 & $\mathrm{C}$ \\
\hline SDSS J0140+2344 & -187.99 & 1.69 & 6561.38 & $\mathrm{C}$ \\
\hline SDSS J0140+2344 & -202.03 & 0.82 & 6824.57 & $\mathrm{C}$ \\
\hline SDSS J1422+0031 & -125.43 & 2.26 & 6717.50 & $\mathrm{~S}$ \\
\hline SDSS J1422+0031 & -113.96 & 2.32 & 7071.59 & $\mathrm{~S}$ \\
\hline SDSS J1422+0031 & -112.35 & 0.23 & 7122.52 & $\mathrm{~S}$ \\
\hline
\end{tabular}


Appendix B: Compilation of 45 CEMP stars with multiple radial velocity measurements

Table B.1. List of 45 CEMP-no stars with multiple radial velocity measurements.

\begin{tabular}{|c|c|c|c|c|c|c|c|c|c|c|c|c|c|}
\hline Name & $n_{\mathrm{rv}}$ & $\begin{array}{r}r v_{\mathrm{w}} \\
\left(\mathrm{km} \mathrm{s}^{-1}\right)\end{array}$ & $\begin{array}{r}\sigma_{\mathrm{r} v} \\
\left(\mathrm{~km} \mathrm{~s}^{-1}\right) \\
\end{array}$ & $P\left(\chi^{2}\right)$ & Bin? & $\begin{array}{l}T_{\text {eff }} \\
(\mathrm{K})\end{array}$ & $\log g$ & {$[\mathrm{Fe} / \mathrm{H}]$} & {$[\mathrm{C} / \mathrm{Fe}]$} & $\mathrm{A}(\mathrm{C})$ & {$[\mathrm{Ba} / \mathrm{Fe}]$} & {$[\mathrm{Mg} / \mathrm{C}]$} & Ref. \\
\hline $\mathrm{BD}+44^{\circ} 493$ & 61 & -150.09 & 0.63 & 0.015 & 1 & 5430 & 3.4 & -3.83 & 1.35 & 5.95 & -0.60 & -0.89 & 1 \\
\hline BS 16929-005 & 21 & -50.77 & 0.66 & 0.629 & 1 & 5229 & 2.6 & -3.34 & 0.99 & 6.09 & -0.41 & -0.70 & 2 \\
\hline $\mathrm{CD}-24^{\circ} 17504$ & 2 & 136.25 & 0.49 & 0.227 & 1 & 6228 & 3.9 & -3.41 & 1.10 & 6.12 & $<-1.05$ & -0.76 & 3 \\
\hline CS 22166-016 & 9 & -210.39 & 0.77 & 0.607 & 1 & 5250 & 2.0 & -2.40 & $1.02^{a}$ & $7.05^{a}$ & -0.37 & $-0.34^{a}$ & 4 \\
\hline CS 22877-001 & 16 & 166.25 & 0.12 & 0.790 & 1 & 4790 & 1.5 & -3.31 & 1.10 & 6.67 & -0.50 & -1.17 & 5 \\
\hline CS $22878-027$ & 21 & -91.28 & 0.61 & 0.058 & 1 & 6319 & 4.4 & -2.51 & $0.86^{a}$ & $6.78^{a}$ & $<-0.75$ & $-0.97^{a}$ & 2 \\
\hline CS 22949-037 & 26 & -125.74 & 0.29 & 0.889 & 1 & 4630 & 1.0 & -4.38 & 1.16 & 5.97 & -0.60 & -0.36 & 5 \\
\hline CS 22957-027 & 40 & -66.87 & 5.16 & $<10^{-6}$ & 2 & 5220 & 2.7 & -3.19 & 2.61 & 7.87 & -0.81 & -2.55 & 5 \\
\hline CS 29498-043 & 26 & -32.55 & 0.60 & 0.994 & 1 & 4440 & 0.5 & -3.87 & 2.75 & 7.62 & -0.49 & -1.54 & 5 \\
\hline CS 29502-092 & 35 & -67.22 & 0.51 & 0.575 & 1 & 4820 & 1.5 & -3.30 & 1.06 & 6.59 & -1.36 & -1.18 & 5 \\
\hline CS 29527-015 & 6 & 47.13 & 0.42 & 0.988 & 1 & 6577 & 3.9 & -3.32 & 1.18 & 6.29 & & -0.68 & 2 \\
\hline G64-12 & 33 & 442.55 & 1.05 & 0.009 & 1 & 6463 & 4.3 & -3.29 & $1.07^{a}$ & $6.21^{a}$ & -0.07 & $-0.59^{a}$ & 6 \\
\hline G64-37 & 22 & 81.49 & 0.95 & 0.312 & 1 & 6570 & 4.4 & -3.11 & $1.12^{a}$ & $6.44^{a}$ & -0.36 & $-0.74^{a}$ & 6 \\
\hline G77-61 & 13 & -23.94 & 11.35 & $<10^{-6}$ & 2 & 4000 & 5.1 & -4.08 & 2.65 & 7.00 & $<+1.00$ & -2.16 & 7 \\
\hline HE 0020-1741 & 10 & 93.06 & 0.83 & 0.001 & 1 & 4765 & 1.6 & -4.05 & 1.40 & 6.12 & -1.11 & -0.71 & 6 \\
\hline HE 0057-5959 & 3 & 375.64 & 1.60 & 0.143 & & 5257 & 2.7 & -4.08 & 0.86 & 5.21 & -0.46 & -0.35 & 2 \\
\hline HE $0107-5240$ & 7 & 44.78 & 1.91 & $<10^{-6}$ & 2 & 5100 & 2.2 & -5.44 & 3.97 & 7.03 & $<+0.93$ & -3.77 & 8 \\
\hline HE 0219-1739 & 15 & 107.96 & 5.09 & $<10^{-6}$ & 2 & 4238 & 0.5 & -3.09 & 1.90 & 7.55 & $<-1.39$ & & 9 \\
\hline HE $0405-0526$ & 13 & 165.66 & 0.04 & 1.000 & 1 & 5083 & 3.9 & -2.18 & 0.92 & 7.17 & -0.22 & & 9 \\
\hline HE $0557-4840$ & 22 & 211.94 & 0.80 & 0.965 & & 4900 & 2.2 & -4.73 & 1.59 & 5.29 & $<+0.07$ & -1.43 & 10 \\
\hline HE 1012-1540 & 20 & 225.84 & 0.47 & 0.217 & 1 & 5230 & 2.7 & -4.17 & 2.40 & 6.67 & -0.28 & -0.56 & 5 \\
\hline HE 1133-0555 & 9 & 270.70 & 0.34 & 0.810 & 1 & 5526 & 1.3 & -2.40 & 2.20 & 8.31 & -0.58 & & 9 \\
\hline HE $1150-0428$ & 27 & 47.49 & 8.13 & $<10^{-6}$ & 2 & 5208 & 2.5 & -3.47 & 2.37 & 7.35 & -0.48 & -1.98 & 2 \\
\hline HE $1201-1512^{b}$ & 13 & 238.91 & 2.51 & 0.016 & 1 & 5725 & 4.7 & -3.86 & 1.14 & 5.71 & $<+0.05$ & -0.86 & 2 \\
\hline HE 1300-0641 & 2 & 68.79 & 0.11 & 0.162 & 1 & 5308 & 3.0 & -3.14 & 1.25 & 6.54 & -0.82 & -1.23 & 11 \\
\hline HE $1300+0157$ & 20 & 74.40 & 0.70 & 0.871 & 1 & 5529 & 3.3 & -3.75 & 1.31 & 5.99 & $<-0.85$ & -0.98 & 2 \\
\hline HE 1302-0954 & 3 & 32.56 & 0.04 & 0.973 & 1 & 5120 & 2.4 & -2.25 & 1.17 & 7.37 & $<-0.53$ & & 9 \\
\hline HE $1327-2326$ & 17 & 64.38 & 1.30 & 0.202 & 1 & 6180 & 3.7 & -5.71 & 4.18 & 6.90 & $<+1.39$ & -2.63 & 12 \\
\hline HE $1410+0213$ & 23 & 81.12 & 0.18 & 0.614 & 1 & 5000 & 2.0 & -2.14 & 1.92 & 8.21 & -0.26 & -1.81 & 13 \\
\hline HE $1506-0113$ & 29 & -83.50 & 11.22 & $<10^{-6}$ & 2 & 5016 & 2.0 & -3.54 & 1.47 & 6.38 & -0.80 & -0.60 & 2 \\
\hline HE 2139-5432 & 4 & 102.18 & 10.07 & $<10^{-6}$ & 2 & 5416 & 3.0 & -4.02 & 2.59 & 7.01 & $<-0.33$ & -0.99 & 2 \\
\hline HE 2142-5656 & 4 & 103.70 & 0.80 & 0.718 & & 4939 & 1.9 & -2.87 & 0.95 & 6.61 & -0.63 & -0.72 & 2 \\
\hline HE 2202-4831 & 3 & 56.32 & 0.67 & 0.797 & & 5331 & 3.0 & -2.78 & 2.41 & 8.08 & -1.28 & -2.31 & 2 \\
\hline HE 2247-7400 & 2 & 5.78 & 0.59 & 0.602 & & 4829 & 1.6 & -2.87 & 0.70 & 6.58 & -0.94 & -0.69 & 2 \\
\hline HE $2318-1621$ & 7 & -41.77 & 0.28 & 0.218 & 1 & 4846 & 1.4 & -3.67 & 1.04 & 6.30 & -1.61 & -1.34 & 14 \\
\hline SDSS J0140+2344 ${ }^{b}$ & 8 & -198.65 & 4.54 & $<10^{-6}$ & 2 & 5703 & 4.7 & -4.00 & 1.13 & 5.56 & $<+0.34$ & -0.81 & 2 \\
\hline SDSS J0929+0238 & 26 & 388.33 & 10.40 & & 2 & 5894 & 4.5 & -4.97 & $3.91^{a}$ & $7.44^{a}$ & $<+1.46$ & $-3.56^{a}$ & 15 \\
\hline SDSS J1313-0019 & 3 & 255.50 & 13.50 & $<10^{-6}$ & $2 ?$ & 5200 & 2.6 & -5.00 & 2.98 & 6.41 & $<+0.22$ & -2.45 & 16 \\
\hline SDSS J1341+4741 & 5 & -194.68 & 26.63 & $<10^{-6}$ & 2 & 5450 & 2.5 & -3.20 & $0.99^{a}$ & $6.22^{a}$ & -0.73 & $-0.28^{a}$ & 17 \\
\hline SDSS J1422+0031 & 6 & -118.97 & 6.09 & $<10^{-6}$ & 2 & 5200 & 2.2 & -3.03 & 1.70 & 7.11 & -1.18 & -0.95 & 18 \\
\hline SDSS J1613+5309 & 4 & 0.01 & 0.89 & 0.745 & & 5350 & 2.1 & -3.33 & 2.09 & 7.21 & +0.03 & -1.18 & 18 \\
\hline SDSS J1746+2455 & 5 & 78.45 & 0.54 & 0.431 & & 5350 & 2.6 & -3.17 & 1.24 & 6.51 & +0.26 & -0.56 & 2 \\
\hline SDSS J2206-0925 & 3 & 14.83 & 0.90 & 0.626 & & 5100 & 2.1 & -3.17 & $0.64^{a}$ & $5.90^{a}$ & -0.85 & $+0.61^{a}$ & 18 \\
\hline Segue 1-7 & 4 & 204.86 & 0.59 & 0.580 & & 4960 & 1.9 & -3.52 & $2.30^{a}$ & $7.21^{a}$ & $<-0.96$ & $+0.94^{a}$ & 19 \\
\hline SMSS 0313-6708 & 34 & 298.50 & 0.35 & 0.592 & 1 & 5125 & 2.3 & $<-6.50$ & $>5.39$ & 6.02 & & -1.20 & 20 \\
\hline
\end{tabular}

Notes. Columns: star name; number of radial velocity measurements in Table B.2; weighted average of radial velocity measurements in Table B.2; standard deviation of radial velocity measurements in Table B.2; variability criterion $P\left(\chi^{2}\right)$; binary status $(1=$ single, $2=$ binary, labels as collected by Yoon et al. (2016) and updated in this work); effective temperature; surface gravity; iron abundance; carbon-to-iron ratio; absolute carbon abundance; barium-to-iron ratio, magnesium-to-carbon ratio; literature reference. ${ }^{(a)}$ These carbon abundances have not been corrected for evolutionary status (all other measurements have been, as reported in Yoon et al. 2016). However, all of these stars have $\log g \gtrsim 2.0$, where the correction is expected to be small. ${ }^{(b)}$ Of the two provided solutions in the reference, these are the dwarf solutions.

References. (1) Ito et al. (2013), (2) Yong et al. (2013), (3) Jacobson et al. (2015), (4) Giridhar et al. (2001), (5) Roederer et al. (2014), (6) Placco et al. (2016b), (7) Plez \& Cohen (2005), (8) Christlieb et al. (2004), (9) H16a, (10) Norris et al. (2007), (11) Barklem et al. (2005), (12) Frebel et al. (2008), (13) Cohen et al. (2013), (14) Placco et al. (2014), (15) Bonifacio et al. (2015) and for magnesium Caffau et al. (2011), (16) Frebel et al. (2015), (17) Bandyopadhyay et al. (2018), (18) Aoki et al. (2013), (19) Norris et al. (2010), (20) Bessell et al. (2015), and for magnesium Keller et al. (2014). 
Table B.2. Compilation of 710 radial velocity measurements for 45 CEMP-no stars.

\begin{tabular}{lrrrll}
\hline \hline Name & $\begin{array}{r}r v \\
\left(\mathrm{~km} \mathrm{~s}^{-1}\right)\end{array}$ & $\begin{array}{r}\text { Err } \\
\left(\mathrm{km} \mathrm{s}^{-1}\right)\end{array}$ & $\begin{array}{r}\text { HJD } \\
-2450000\end{array}$ & Ref. & Bibcode \\
\hline BD $+44^{\circ} 493$ & -150.66 & 0.55 & -4273.25 & Carney et al. (2003) & 2003AJ...125..293C \\
BD+44 493 & -151.16 & 0.77 & -4034.25 & Carney et al. (2003) & 2003AJ...125..293C \\
BD+44 493 & -149.55 & 0.68 & -3698.25 & Carney et al. (2003) & 2003AJ...125..293C \\
BD $+44^{\circ} 493$ & -151.57 & 0.57 & -3662.25 & Carney et al. (2003) & 2003AJ...125..293C \\
BD+44 493 & -150.93 & 0.67 & -3634.25 & Carney et al. (2003) & 2003AJ...125..293C \\
$\ldots$ & $\ldots$ & $\ldots$ & $\ldots$ & $\ldots$ & $\ldots$ \\
\hline
\end{tabular}

Notes. We do not include Gaia DR2 radial velocities in this table because they are averages over multiple measurements in time. This table is available in its entirety at the CDS.

\section{Appendix C: Additional figures}

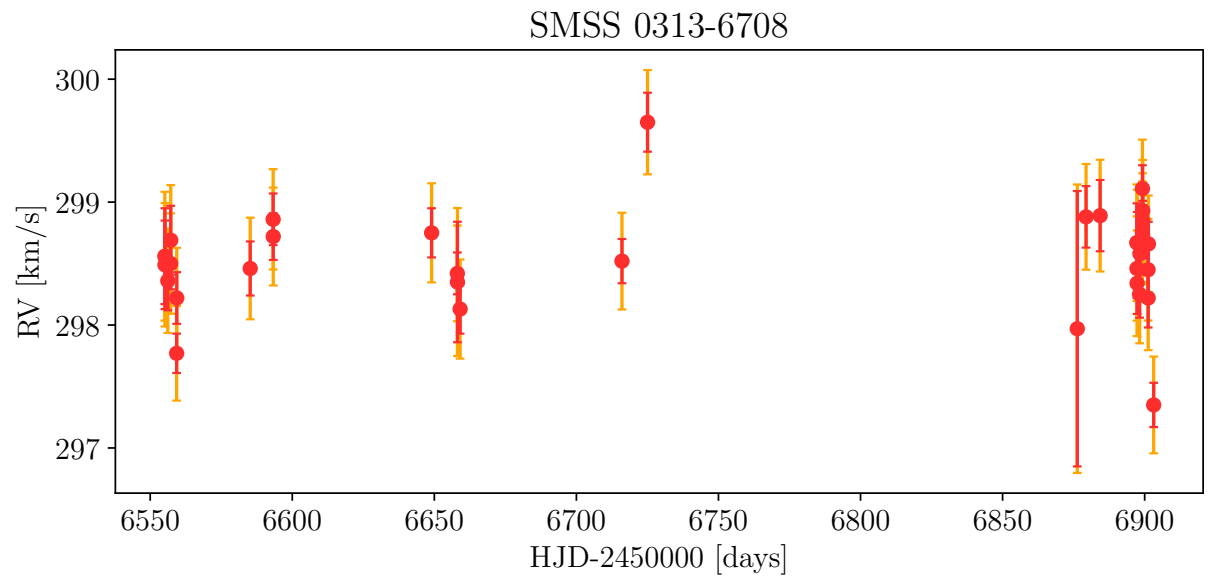

Fig. C.1. Radial velocities for SMSS 0313-6708, derived from archive UVES spectra. The red error bars represent the uncertainties coming from FXCOR, and the orange error bars include an uncertainty floor of $0.35 \mathrm{~km} \mathrm{~s}^{-1}$. 
A. Arentsen et al.: Binarity among CEMP-no stars: an indication of multiple formation pathways?
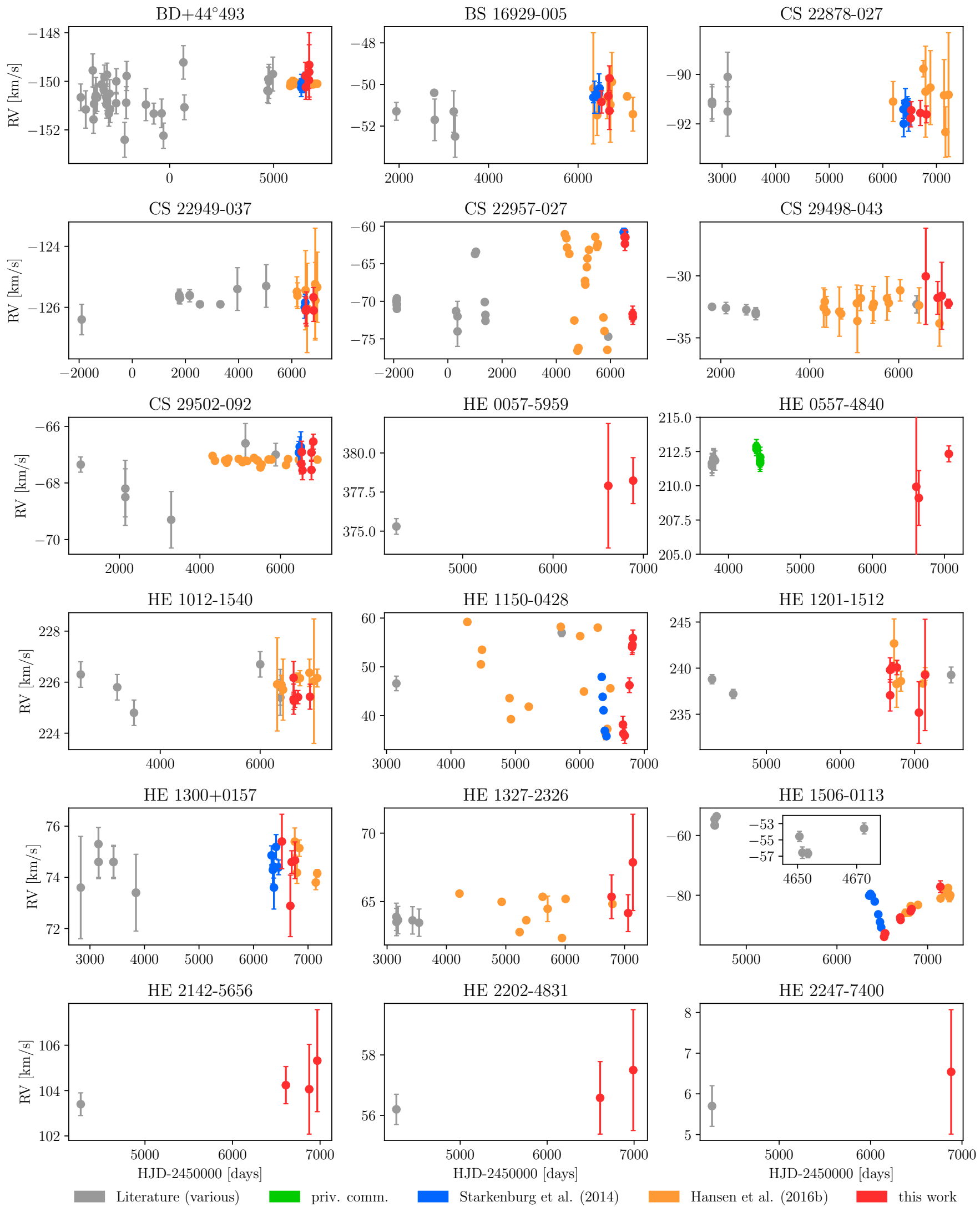

Fig. C.2. Radial velocities of the 18 program stars that have not yet been shown in the main text, ordered alphabetically. For seven measurements of HE 1327-2326 reported by Hansen et al. (2016b), no uncertainties are provided. 